\title{
Stereoscopic Mechanisms in Monkey Visual Cortex: Binocular Correlation and Disparity Selectivity
}

\author{
Gian F. Poggio, Francisco Gonzalez, ${ }^{a}$ and Friedemann Krause ${ }^{\mathrm{b}}$ \\ Bard Laboratories of Neurophysiology, Department of Neuroscience, The Johns Hopkins University School of Medicine, \\ Baltimore, Maryland 21205
}

\begin{abstract}
The neural signals in visual cortex associated with positional disparity and contrast texture correlation of binocular images are the subject of this study. We have analyzed the effects of stereoscopically presented luminous bars and of dynamic random-dot patterns on the activity of single neurons in cortical visual areas V1, V2, and V3-V3A of the alert, visually trained rhesus macaque. The interpretation of the results and considerations of possible neural mechanisms led us to recognize 2 functional sets of stereoscopic neurons. (1) A set of neurons, tuned excitatory (TO) or tuned inhibitory (TI), which respond sharply to images of zero or near-zero disparity. Objects at or about the horopter drive the TO neurons and suppress the $\mathrm{Tl}$, while objects nearer and farther have the opposite effects on each type, inhibition of the TO and excitation of the TI. The activity of these neurons may provide, in a reciprocal way, the definition of the plane of fixation, and the basic reference for binocular single vision and depth discrimination. (2) A second set of neurons includes tuned excitatory at larger crossed or uncrossed disparities (TN/TF) and neurons with reciprocal excitatory and inhibitory disparity sensitivity with cross-over at the horopter (NE/FA). Binocularly uncorrelated image contrast drives these neurons to a maintained level of activity, which shifts, in response to correlated images, toward facilitation or suppression as a function of positional disparity. These neurons may operate in the neural processing leading to stereopsis, both coarse and fine, and also provide signals for the system controlling binocular vergence. These results indicate that cortical visual neurons are binocularly linked to respond to the relative position and contrast of the images over their receptive fields, and also that both these aspects of binocular stimulation may be utilized by the brain as a source of stereoscopic information.
\end{abstract}

\footnotetext{
Received Aug. 13, 1987; revised Apr. 7, 1988; accepted May 13, 1988.

This research was supported by Grant 5 R01 EY02966 from the National Institutes of Health, USPHS, and by a grant from the A. P. Sloan Foundation. F.G. was a Postdoctoral Fellow in Neuroscience, supported by the Fulbright/MEC Research Program (1984-1985) and by the NIH/Fogarty International Center (1985-1986). F.K. was a Visiting Scientist in Neuroscience supported by Grant 1 P01 NS20868 from the NIH, USPHS (1984-1985).

Correspondence should be addressed to Gian F. Poggio, The Johns Hopkins University School of Medicine, 725 North Wolfe Street, Baltimore, MD 21205.

${ }^{a}$ Present address: Department of Physiology, University of Santiago, Santiago de Compostela, Spain.

b Department of Biophysics, Philipps-University, D-3550 Marburg, Federal Republic of Germany.

Copyright (C) 1988 Society for Neuroscience $0270-6474 / 88 / 124531-20 \$ 02.00 / 0$
}

The brain utilizes the signals from the 2 eyes to recover the relative depth of objects. Wheatstone (1838) first demonstrated that a difference, or disparity, in the relative horizontal position of the object's images in the 2 eyes can generate an impression of depth and solidity. Julesz $(1960,1971)$ later showed by means of random-dot stereograms that binocular disparity is the sufficient cue for stereoscopic depth perception. Physiological evidence indicates that a substantial number of neurons in the striate cortex (V1) of the alert macaque signal the horizontal positional disparity of retinal images (Poggio and Fischer, 1977; Poggio, 1980, 1984; Poggio and Talbot, 1981; Poggio et al., 1985b). Disparity-sensitive neurons have been identified in all extrastriate cortical visual areas of the macaque, from V2 to V5, and in even higher proportion than in VI (Hubel and Wiesel, 1970; Poggio and Fischer, 1977; Maunsell and Van Essen, 1983; Poggio, 1984, 1985; Poggio et al., 1985a, b; Burkhalter and Van Essen, 1986; Felleman and Van Essen, 1987; Hubel and Livingstone, 1987). We refer to these neurons collectively as "stereoscopic" neurons on the conjecture that their response reflects the early cortical processing leading to binocular depth perception.

During normal vision, left and right receptive fields of a single neuron superimpose in visual space at different distances from the eye as a function of their relative positions in the field of view, i.e., receptive field disparity (Barlow et al., 1967; Joshua and Bishop, 1970). Thus, receptive fields that occupy topographically corresponding parts of the 2 retinas, that is, fields with zero disparity, will superimpose at the distance of convergent fixation (at the horopter), whereas pairs of receptive fields that occupy noncorresponding retinal regions will superimpose at nearer or farther distances, on opposite sides of the horopter. Accordingly, the pair of receptive fields of a single cortical neuron sometimes will "see" the identical, or nearly identical, concurrent images of a single object in depth but more often, when no object is present at the depth location of their binocular overlap, the 2 receptive fields will "see" images of different objects.

The response properties of visual neurons, from retina to cortex, are determined by the relation between the configurational aspects of the visual image and the organization of the neuron's receptive field. Cortical visual neurons are typically binocular and receive an input from both eyes through receptive fields of similar organization (Hubel and Wiesel, 1968, 1977; Schiller et al., 1976b; Maske et al., 1984; Skottun and Freeman, 1984). The stimulus conditions that evoke uniquely binocular responses - the binocular stimuli-rest on the relations between images and receptive fields in the 2 eyes. "Positional disparity" 
describes the relative position of an object's images in the 2 eyes as determined by the geometry of binocular vision. Positional disparity is zero when left and right images occupy topographically corresponding positions in each retina. Following our convention (Poggio and Fischer, 1977), disparities associated with objects farther than the distance of fixation are given positive values (uncrossed disparities), and those associated with objects nearer than fixation are given negative values (crossed disparities). Various aspects of the images, other than position, may have the same or different binocular configuration (temporal, textural, chromatic, etc.), not all necessarily contributing to the stereoscopic process (Livingstone and Hubel, 1987). "Image correlation" describes the relation between the configuration of the images in the 2 eyes. In this paper, it refers strictly to the luminous contrast texture of dynamic random-dot patterns, either the same in the 2 eyes (image correlation) or not (image uncorrelation). Clearly, position and texture are operationally interacting aspects of binocular stimulation, and positionally disparate stimuli are uncorrelated for neurons with topographically corresponding fields and corresponding stimuli are uncorrelated for neurons with disparate fields. We shall speak of "binocular correlation" and "binocular uncorrelation" to indicate the conditions in which identical images occupy - or do not-spatially matched receptive fields in the 2 eyes.

We have attempted to further our understanding of the dynamics of operation of cortical stereoscopic neurons by identifying the components of the neural signal associated with image correlation and those associated with positional image disparity. Observations were made in alert, visually trained rhesus macaques; disparity sensitivity of single cortical neurons was assessed with a luminous bar whose configuration was adjusted to evoke "optimal" responses; correlation sensitivity was tested with dynamic random-dot stereopairs with identical or mismatched dot patterns delivered to the 2 eyes. We found that a substantial proportion of neurons in the visual cortex of the macaque monkey, increasing in number from $\mathrm{V} 1$ to $\mathrm{V} 2$ and V3-V3A, signal by their impulse activity the direction and magnitude of horizontal retinal disparity of binocular images (disparity sensitivity). Moreover, many cortical neurons in the 3 areas give differential responses, excitatory and inhibitory, to the "same" and the "not-same" contrast texture of the images over their receptive fields in the 2 eyes (correlation sensitivity). Neurons with these stereoscopic properties are thought to provide the basic signals for binocular depth vision. Preliminary findings have been presented elsewhere (Poggio el al., 1985a; Gonzalez et al., 1986).

\section{Materials and Methods}

Experiments were made in male Macaca mulatta weighing 3-5 kg. The monkeys were trained to execute a fixation/detection task in an apparatus in which the animal's left and right eyes saw separate display screens. A PDP1 1/34A minicomputer was used to regulate and monitor the animal's behavioral task, to control visual stimulation, and to collect behavioral and electrophysiological data (Poggio and Talbot, 1981). A behavioral trial began with presentation of the fixation target, was continued if the animal closed a signal key within a few hundred milliseconds, and was terminated if the animal released the key within a limited time after a change in target configuration. Each correct detection was rewarded with a drop of water. Criteria of $90 \%$ correct detections or better were reached by successive approximations, and trained animals performed 1000-1300 trials during a recording session of 4-6 hr. Stereoscopic and monocular test stimuli were delivered during the periods of maintained eye fixation. They were used to determine the functional properties of cortical visual neurons; they did not control behavior as the target did.
Two to three weeks before beginning the recording experiment, a head-holding device was cemented to the skull in an aseptic surgical procedure under pentobarbital anesthesia. Final training was conducted with the head fixed, and on its completion a short, cylindric stainless steel chamber (10 mm diameter) was mounted over the visual cortex of the left hemisphere under general anesthesia. Daily recording sessions started several days later, when the animal had recovered from surgery. The electrical signs of the impulses discharged by cortical neurons were recorded from extracellular positions with platinum/iridium glass-coated microelectrodes (Wolbarsht et al., 1960) carried by a Chubbuck microdrive and inserted via the oil-filled, hydraulically closed chamber and through the intact dura into the occipital cortex. Eye position was routinely monitored with an infrared corneal reflex system (Motter and Poggio, 1984). When thickening of the dura mater made successful microelectrode penetrations unlikely ( $3-4$ weeks), the experiment was continued on the right hemisphere after mounting a new recording chamber over the visual cortex.

Visual stimulation. Fields of dynamic random dots were generated on 2 CRT displays (Hewlett-Packard, model 1311) at 67 frames/sec (15 msec frametime). The positions of the dots on the 2 screens were controlled by a computer-driven digital display generator (Julesz et al., 1976). The display was constructed as a $100 \times 100$ dot raster subtending $10^{\circ}$ at the monkey's eye $\left(0.1^{\circ}\right.$ interdot separation, $\left.100 \mathrm{dots} / \mathrm{deg}^{2}\right)$. It was made up of "bright" (intensified) and "dark" pixels in different relative proportions. The bright/dark dot density could be selected, and the mean luminance of the display was linearly related to dot density between 0.6 $\mathrm{cd} / \mathrm{m}^{2}$ (bright dot density $=1 \%$ ) and $14.4 \mathrm{~cd} / \mathrm{m}^{2}$ (bright dot density $=$ $100 \%$ ). The luminance of the blank screen was not measurable with our detector.

During the course of the electrode penetration, binocular and monocular stimuli were presented within the monkey's field of view during the periods of maintained eye fixation. Frequent determinations of the response properties of the neurons encountered were made by listening to and visually monitoring the ongoing activity, while moving under manual control a luminous bar of $100 \%$ dot density, adjusted in size and orientation for each neuron to evoke the subjectively judged "optimal" binocular response. The 2-dimensional (2D) location of this response was taken as the center of the "response field," and for cach field location, the fixation target was shifted to a new position such that the current response field was centered on the center of the CRT display. Field location was repeatedly checked on the unresolved multiunit activity of small clusters of cells and on the impulse activity of each isolated neuron. A coarse determination of the size of the response field was also made for a number of cells. Together with the response field topography, certain $2 \mathrm{D}$ properties of the neurons (orientation and direction selectivities) and ocular dominance were routinely assessed and recorded.

Two aspects of the 3-dimensional properties of cortical neurons were evaluated: horizontal disparity selectivity and binocular correlation sensitivity. (1) Positional disparity selectivity was determined with moving or flashing "optimal" bars presented stereoscopically (solid figure stereograms) against a blank or a random-dot background (Poggio and Talbot, 1981). For each neuron in this study a disparity selectivity profile was constructed as described below (see Data analysis). For many of these neurons, the responses to dynamic random-dot stereograms (Julesz, 1960, 1971) were also recorded. (2) Image correlation sensitivity was assessed with dynamic random-dot stereopairs (correlograms) (Julesz et al., 1976; Tyler and Julesz, 1976, 1978) in which the random pattern of dots within a test area centered over the neuron's binocular receptive field was made to alternate from identical in the 2 eyes (correlation) to different (uncorrelation). Test areas of the size and orientation of the selected bar were used, but the neuron's sensitivity was usually evaluated with larger square or rectangular patterns $\left(1.0^{\circ}-4.0^{\circ}\right.$ side); the background subtended $10^{\circ}$ and was seen identically by both eyes. The binocularly uncorrelated central test areas were produced in 2 ways. The first method used synchronized and independent random-dot sequences of identical dot densities, one sequence for the left screen, one for the right. The relative densities of bright and dark dots determine the average luminance of the display and govern the average number of spatially corresponding and noncorresponding dots seen by the 2 eyes. The second method employed synchronized sequences of $50 \%$ dot density, the left and the right dot sequences each being the complement of the other: Each bright dot in one eye is matched with a dark dot in the other eye (anticorrelation or negative correlation). This condition of stimulation is illustrated in Figure 1, where the correlated and anticorrelated test areas are outlined for emphasis with a white line. The white 


\section{image correlation}
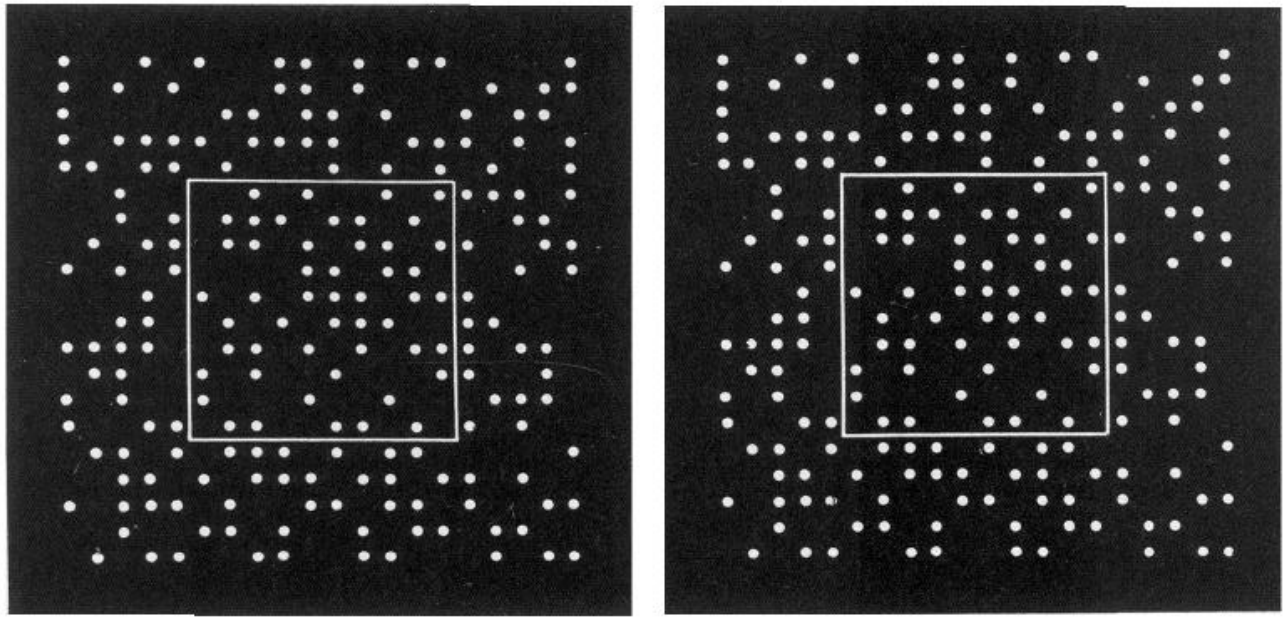

\section{LEFT EYE}
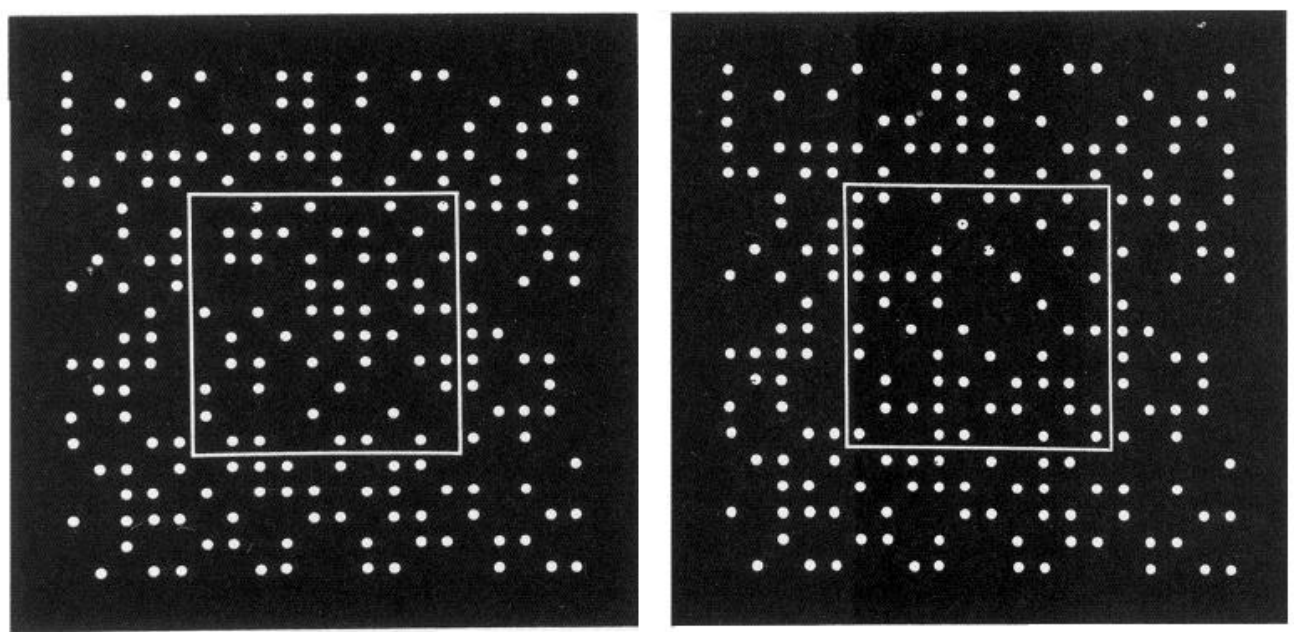

\section{image anticorrelation}

Figure 1. Schematic illustration of random-dot correlograms. The monkey, its head firmly held, views 2 display screens ( $L E F T E Y E, R I G H T E Y E)$ separately with each eye. Fields of dynamic random dots were generated on the 2 screens and binocular correlograms were produced by making the dot patterns over a central area on each screen different from each other, either by using 2 independent random-dot sequences or by switching the pattern on one screen to be the complement of the pattern on the other screen. The stereofields shown in this figure illustrate this latter condition, with the test area outlined for emphasis by a white line, which, of course, did not appear on the display: In $A$, the dot patterns seen by the left and right eyes are identical (image correlation). In $B$, the patterns over a central square of the stereofields are the complement of each other (image anticorrelation); the surrounding background remains binocularly correlated. Neural sensitivity for binocular correlation is tested by alternating uncorrelated central stereo images over the neuron's receptive fields several times during the period of maintained eye fixation. line did not, of course, appear on the display. Nearly all neurons were tested with stereopatterns of anticorrelation (mean luminance $7.0 \mathrm{~cd} /$ $\mathrm{m}^{2}$ ), as well as with randomly uncorrelated fields, with the density of bright dots from 1 to $50 \%$.

Human subjects who look at uncorrelated patterns in our apparatus generally experience a compelling illusion of depth, the uncorrelated square seen usually "near," in front of the surrounding correlated background. When horizontal disparity, whether crossed or uncrossed, is introduced between the 2 uncorrelated squares, the subject sees the square become wider and "rectangular" in appearance, suggesting that left and right fields are not fused but superimposed. This is confirmed by the fact that when the uncorrelated dot patterns of 2 disparate squares are made identical (correlated), the square again is seen "square" and nearer or farther than the background depending on the sign of the disparity. A similar phenomenon has been described by O'Shea and Blake (1986)

Data analysis. Positional disparity sensitivity was evaluated for each neuron with luminous bars moving in frontoparallel paths with various degrees of horizontal disparity and/or with stationary flashing stimuli as well. Plots of the binocular responses evoked by a series of stimuli with crossed, zero, and uncrossed disparities provided a disparity selectivity profile for the neuron. Estimates of the average magnitude of the neural response and of its variability were made from impulse counts and expressed as impulses/sec. These estimates were obtained from at least 10-20 responses collected during the course of 4-6 successive fixation trials. For each test series, a single counting period bracketing the response maximum was used with its time position and duration (200-500 msec) selected by visual inspection of rasters of the impulse sequences, and frequently also from poststimulus time histograms. Differential responses of single neurons to binocular correlation and uncorrelation were usually strong and unambiguous, and qualitative estimates of excitation or inhibition could be made directly from visual inspection of the impulse sequences.

Microelectrode penetrations through the visual cortex: recording characteristics. Microelectrode penetrations were made in the dorsal striate cortex and underlying extrastriate cortex and were directed into the occipital lobe with an oblique approach nearly perpendicular to the cortical surface, about $45^{\circ}$ from the sagittal plane and $45^{\circ}$ behind the vertical. The penetrations were conducted by slowly advancing (withdrawing) the recording probe while continuously listening to and visually monitoring the ongoing neural activity. Frequent notations were made of the properties of the neural recording and of the associated electrode depth (micrometer). The first recorded event in the penetration is the large drop in electrical impedence that occurs when the electrode's tip leaves the oil-filled chamber to make contact with the dura mater. Next, an attempt is made to identify the top of V1 by detecting signs of neural activity, both unitary spikes and slow waves. As the electrode advances through the striate cortex, it is usually possible to recognize, about 1000 $\mu \mathrm{m}$ below the estimated cortical surface, a region of dense multiunit activity discharging in a sustained fashion, not orientation selective, and 
Table 1. Number of neurons studied in each area of each monkey that were sensitive to positional disparity $(D S P)$ and to binocular image correlation ( $C O R$ )

\begin{tabular}{|c|c|c|c|c|c|}
\hline \multirow[b]{2}{*}{ Area } & \multirow[b]{2}{*}{$\begin{array}{l}\text { Mon- } \\
\text { key }\end{array}$} & \multicolumn{2}{|c|}{$\begin{array}{l}\text { Disparity } \\
\text { sensitivity }\end{array}$} & \multicolumn{2}{|c|}{$\begin{array}{l}\text { Correlation } \\
\text { sensitivity }\end{array}$} \\
\hline & & $\begin{array}{l}\text { DSP/ } \\
\text { TOT }\end{array}$ & $\begin{array}{l}\% \\
\text { DSP }\end{array}$ & $\begin{array}{l}\text { COR/ } \\
\text { TOT }\end{array}$ & $\begin{array}{l}\% \\
\mathrm{COR}\end{array}$ \\
\hline \multirow[t]{7}{*}{ V1 } & 0.14 & $24 / 66$ & 36 & $15 / 41$ & 36 \\
\hline & 0.31 & $28 / 70$ & 40 & $22 / 47$ & 46 \\
\hline & 0.59 & $57 / 131$ & 44 & $19 / 66$ & 29 \\
\hline & 0.63 & $29 / 59$ & 49 & $11 / 34$ & 32 \\
\hline & 0.25 & $37 / 75$ & 49 & $17 / 52$ & 33 \\
\hline & 0.41 & $34 / 64$ & 53 & $18 / 31$ & 58 \\
\hline & 0.07 & $20 / 37$ & 54 & $12 / 24$ & 50 \\
\hline Total & & $229 / 502$ & & $114 / 295$ & \\
\hline \multirow[t]{2}{*}{$\mathrm{V} 2$} & 0.41 & $34 / 52$ & 65 & $11 / 21$ & 52 \\
\hline & 0.07 & $27 / 42$ & 64 & $17 / 33$ & 51 \\
\hline Total & & $61 / 94$ & & $28 / 54$ & \\
\hline \multirow[t]{2}{*}{ V3-V3A } & 0.41 & $40 / 52$ & 77 & $14 / 26$ & 54 \\
\hline & 0.07 & $11 / 12$ & - & $2 / 6$ & - \\
\hline Total & & $51 / 64$ & & $16 / 32$ & \\
\hline Grand total & & $341 / 660$ & $(51.7)$ & $158 / 381$ & $(41.5)$ \\
\hline
\end{tabular}

driven by stimulation of one eye and not the other. This location is in layer IVc (Poggio et al., 1977). After traversing the infragranular layers of V1, often characterized by strong and consistent responses to visual stimulation, the electrode enters the underlying white matter; all cellular activity subsides and the record becomes flat. The thickness of the active region just traversed (V1) was usually 1500-1600 $\mu \mathrm{m}$. When moving through white matter, the electrode recorded from time to time the characteristic initially positive spikes of isolated single fibers and, after a gap of $400-600 \mu \mathrm{m}$, reached the deep layers of area V2 in the posterior bank of the lunate sulcus. The extent of this second region of cellular activity varied from penetration to penetration. It was still present after $3000-4000 \mu \mathrm{m}$ of travel when the penetration passed through the fold of the cortex at the fundus of the lunate sulcus. More frequently, however, area V2 activity extended some 1000-1200 $\mu \mathrm{m}$; beyond this, the record became again flat and unresponsive. The width of this second gap varied over a wide range, but in general after $500-1000 \mu \mathrm{m}$ of silence an unresolved multiunit activity and single neuron impulses could be recorded once again and visual responses could be readily evoked. Correlation between the anatomical reconstructions and the physiological landmarks encountered during the penetration indicated that this cellular region was the cortex of the anterior bank of the lunate sulcus, and thus likely to include areas V3 and V3A (see Results).

Anatomical identification of microelectrode penetrations. On the day after the last recording session, the monkey was anesthetized with sodium pentobarbital injected intravenously and a number of reference pins (3-6) inserted into the brain at selected coordinates in each recording chamber, and in a direction parallel to that of the microelectrode penetrations. For the 2 "principal" monkeys of this study (see Results), the animal was perfused with $0.9 \%$ PBS followed by $4 \%$ paraformaldehyde and $0.1 \%$ glutaraldehyde in $0.15 \mathrm{~m}$ phosphate buffer. For the 5 "supporting" monkeys the brain was not perfused but was removed from the skull and fixed in $10 \%$ phosphate-buffered formalin for several days. The brains of all animals were blocked and embedded in celloidin; serial sections were cut at $20 \mu \mathrm{m}$, mounted, and stained with thionine $1 \%$.

Immediately after perfusion or death of the animal, the brain was exposed, and photographs were taken of the region of cortex explored, with the plane of the film perpendicular to the reference pins. A surface map of the points of entry of the microelectrodes into the cortex was obtained for each hemisphere by projecting a template of the micropositioner coordinates, scaled and appropriately oriented, onto enlarged photographs $(10 \times)$ of the brain surface. From measurements of the distance between reference pins on the surface map and on the histological sections, as well as of measurements of the position within the cortex of the recognizable electrolytic lesions made during the penetra- tions, shrinkage factors were calculated for each block of brain, separately for the 3 dimensions. For the 4 hemispheres of the 2 "principal" monkeys, average total shrinkage was 23,28 , and $30 \%$ ( 2 blocks).

In our experience, electrolytic lesions made more than 3 weeks before the death of the animal are difficult to identify in thionin-stained sections. Moreover, the distortion of tissue during embedding, and especially the irregular widening of sulci, make measurements of depth in infolded cortex uncertain. All of the electrolytic lesions we were able to recognize were used in our reconstructions, but the identification of the position and direction of the penetrations in the brain was in general derived indirectly. First, the sections deemed to contain each penetration were identified from measurements made on the surface map. Then, the location of the penetration was calculated and marked on drawings of the sections. Finally, estimates of the location of the recording sites within the brain were obtained by correlation of the anatomical measurements with the physiological landmarks and functional properties that could be identified during the recording session.

\section{Results}

The neuron sample for this study was obtained from 2 "principal" monkeys in which areas V1, V2, and V3-V3A were studied in the same animal, frequently in the same penetration, and from 5 "supporting" monkeys in which only the properties of neurons in area V1 were investigated. In the 2 principal monkeys, 83 penetrations were made in area $V 1$ of 4 hemispheres; 61 of these penetrations extended to area V2, and 30 reached the anterior bank of the lunate sulcus (V3-V3A). Certain basic $2 \mathrm{D}$ response properties (response field location, ocularity, orientation, and direction sensitivities) were qualitatively determined for several hundred neurons in area V1, for 172 in V2, and 118 in V3-V3A. A subset of these neurons was tested for the stereoscopic properties of disparity sensitivity and of correlation sensitivity: 101 and 55 in V1, 94 and 54 in V2, and 64 and 32 in V3-V3A, respectively. In the other 5 monkeys, 180 penetrations were made in $\mathrm{V} 1$; disparity selectivity was determincd for 401 neurons and correlation sensitivity for 240 of them. Observations made in some of these 5 animals have been described, in part, in previous publications (Poggio, 1984; Poggio et al., 1985b)

All response properties that we have analyzed, whether $2 \mathrm{D}$ properties (orientation and direction selectivities) or 3D properties (disparity and correlation sensitivities) were qualitatively similar in all cortical areas explored and in all monkeys studied. On the other hand, the frequency of occurrence of stereo properties in V1 varied considerably from monkey to monkey, even though topographically corresponding regions of striate cortex were explored in all animals. For this reason, we have analyzed the 3 areas of interest in the same animal, and numerical comparisons between neurons' properties in V1, V2, and V3-V3A will be restricted to the 2-monkey group. Table 1 shows the number of disparity-sensitive neurons (DSP) and of correlation sensitive neurons (COR) that were tested for cach monkey in each region of cortex.

\section{Identification of cortical area of recording}

The anatomical reconstruction of the site and direction of the penetrations, the alternations of cortical and extracortical regions traversed by the electrode, and the "response field sequence" encountered make it possible to assign neurons to areas with some degree of certainty. Area V1 on the exposed surface of the occipital lobe is easily recognized by its superficial location, topographic organization, and functional properties $\mathrm{Hu}$ bel and Wiesel, 1968; Poggio et al., 1977; Dow et al., 1981, 1985; Van Essen et al., 1984). Area V2 is adjacent to V1 and, in the part of brain we have explored, occupies the medial 


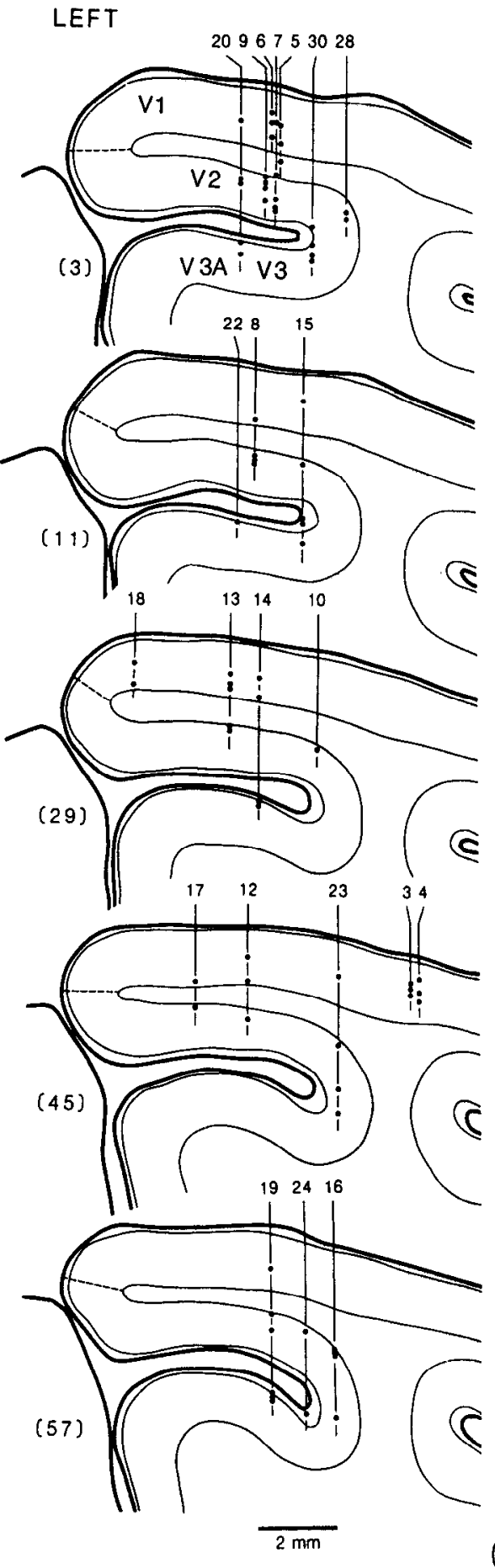

$(41-V)$
RIGHT
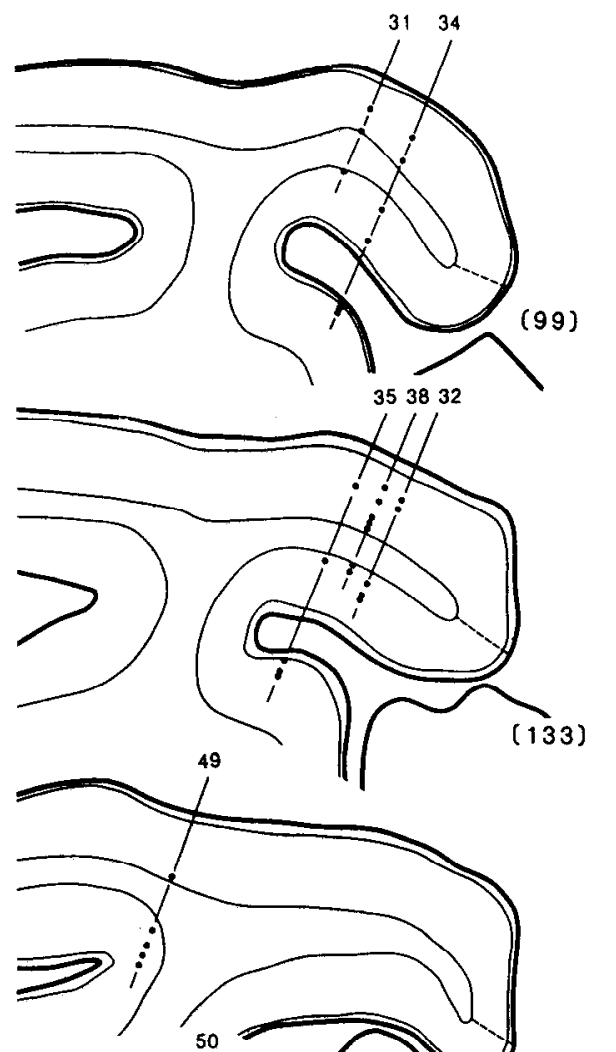

(211)

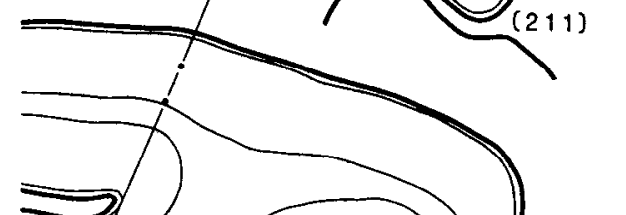

Figure 2. Anatomical reconstruction of microelectrode tracks in the brain of monkey $41-\mathrm{V}$. The positions of each penetration and of the nuerons quantitatively studied within it are shown. They were estimated on the basis of several criteria: location of the point of entry at the surface of the cortex, physiological landmarks (see text), and electrolytic lesions and track marks, generally found only in the hemisphere explored last (right). Twenty-two out of 30 penetrations made in the left hemisphere were successfully reconstructed (79 neurons), and 10 out of 20 in the right hemisphere (56 neurons). These track reconstructions provide only a relative measure of the depth at which the neurons were isolated in each cortical area, but they are reliable estimates for the identification of the general position and of the area in which the neuron was located. The number above each line representing the track is the penetration's serial number; the number in brackets at the side of each section is the number of the section. surface of the occipital operculum, forming the posterior bank of the lunate sulcus (Cragg, 1969; Zeki, 1969; Baizer et al., 1977; Van Essen and Zeki, 1978; Lund et al., 1981; Gattass et al., 1981; Van Essen et al., 1982, 1986). Neurons in area V2 have receptive fields larger and more peripherally located than those in the overlying V1, often at similar elevations. In the deeper cellular region (see Materials and Methods), the response field shifts several degrees away from the fields in V2, generally but not always more eccentrically; the field's size becomes larger and its extent is often difficult to define. The topography of this region tends to be irregular and coarse, and the location of the fields of successively isolated neurons may shift unpredictably, often within short distances of microelectrode travel. Figure 2 shows the reconstruction of the sites of recording for left and right hemispheres of one monkey (41-V). Figure 3 depicts 2 representative penetrations together with maps of the positions of the response fields of cells successively isolated in the 3 cellular regions.

Neurons isolated in the deeper region were located in the cortex of the anterior bank of the lunate sulcus. This region of cortex is the lateral component of the third visual complex of Zeki (1978a, b) and includes 2 neighboring visual areas, V3 and 


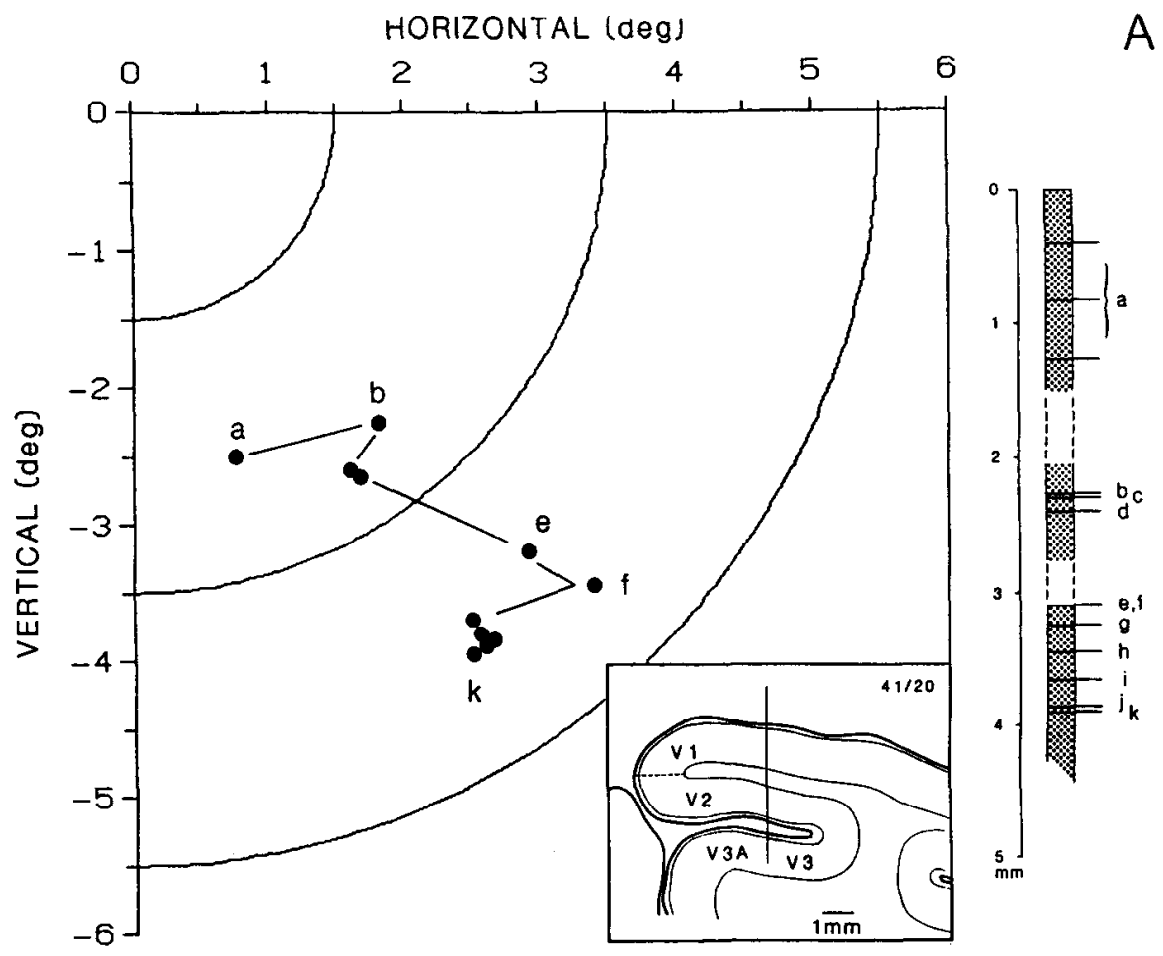

Figure 3. Reconstruction of 2 representative penetrations in V1, V2, V3$\mathrm{V} 3 \mathrm{~A}$ in the left $(A)$ and right $(B)$ hemispheres of monkey $41-\mathrm{V}$, as outlined in the insets. Measurements along the electrode tract are shown on the right: The stippled segments indicate regions of cellular activity, and clear segments the regions identified as white matter. Each horizontal tick marks the depth at which a neuron was isolated and the location of its receptive field's center defined. The lower case letters identify each of the fields with a different location or a group of fields with the same location (VI). The receptive fields were located in the lower visual field and their centers positions are plotted marked with dots in the middle of the figure. The "response field sequence" for the penetration is shown by connecting the field center positions with continuous line segments.

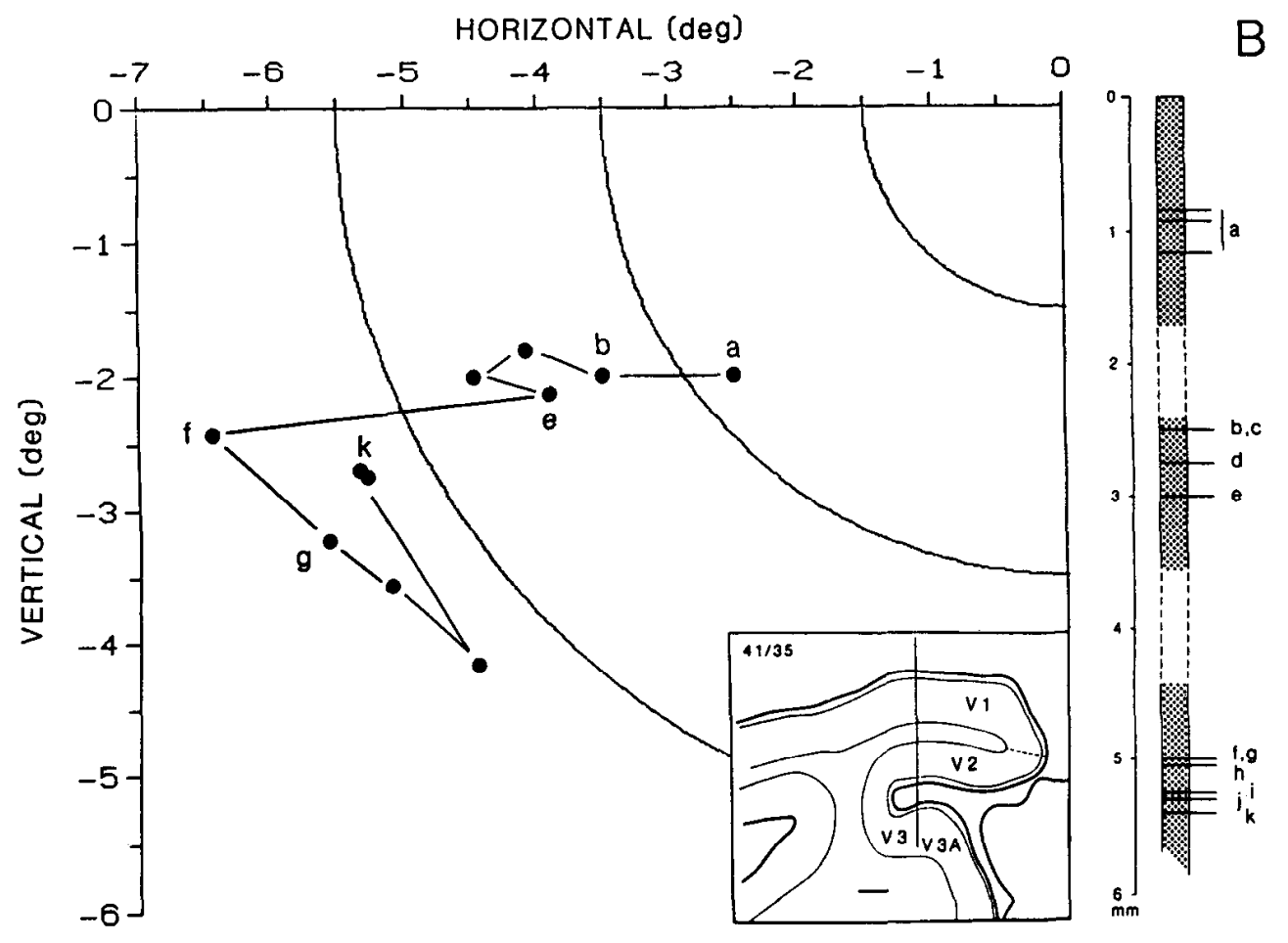

V3A, the former medial and posterior to the latter (Cragg, 1969; Zeki, 1978a, b; Van Essen and Zeki, 1978; Van Essen et al., 1986; Felleman and Van Essen, 1987). Anteriorly, area V3A borders with area V4; posteriorly, area V 3 extends to the fundus of the lunate sulcus, where it adjoins area V2. The ventral vertical meridian is represented along the V3/V3A border and the horizontal meridian along the V2/V3 boundary (Zeki and Sandeman, 1976; Zeki, 1978a; Van Essen et al., 1986). There are no cytoarchitectural boundaries between these 2 areas, and their cells have similar functional properties (Zeki, 1978a, b). Area V3, however, can be distinguished from the neighboring cortex because of the presence of heavily myelinated fibers in layers 4-6 (Van Essen et al., 1986; Felleman and Van Essen, 1987). We have not determined the pattern of callosal connections in the brains of our animals, nor have we stained our sections for myelin. Moreover, we have observed no differences in the $2 \mathrm{D}$ or $3 \mathrm{D}$ functional properties of the neurons isolated in this region. Therefore, we have considered the cortex we have 

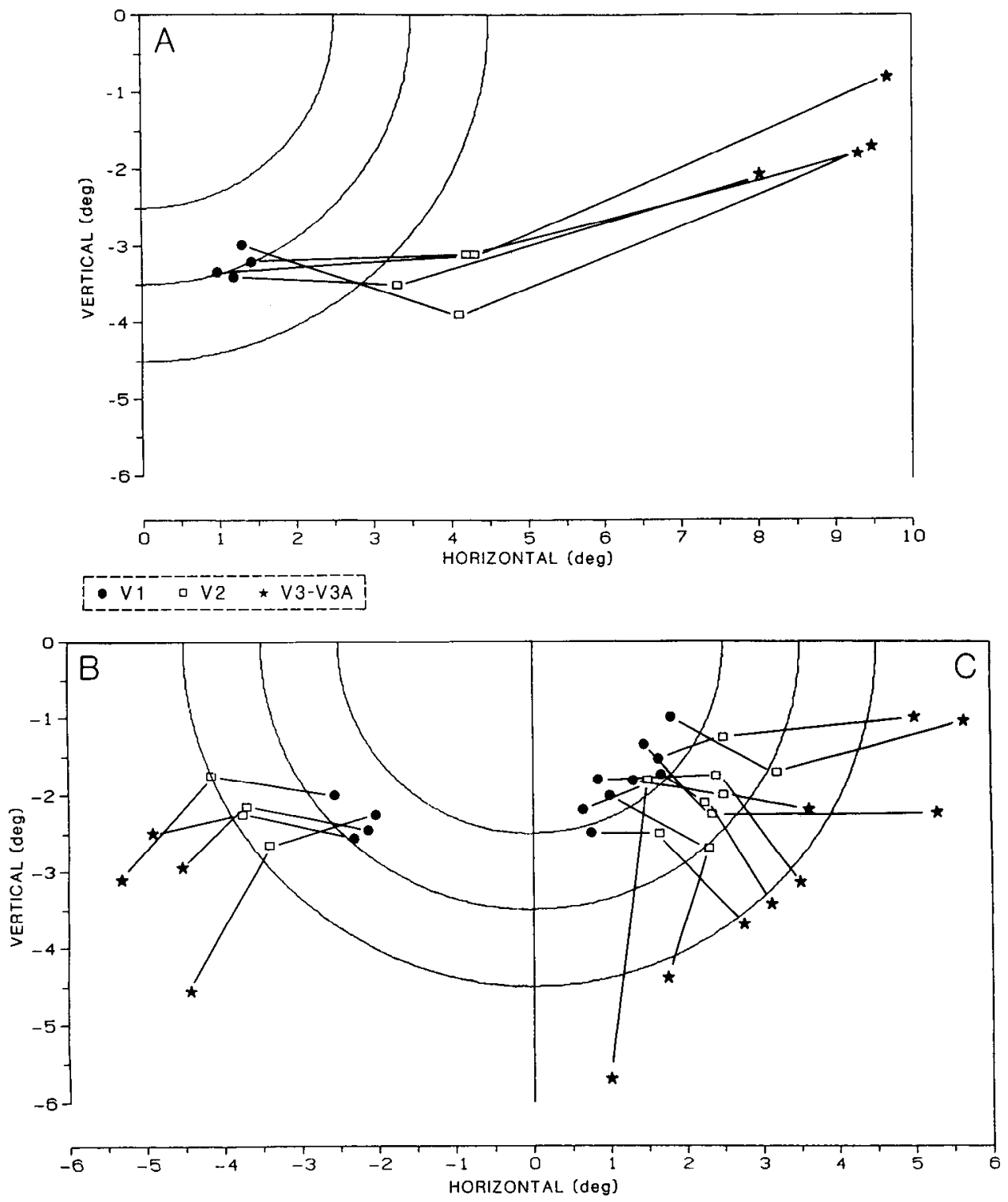

Figure 4. Representation of the "response field sequences" for 3 sets $(A-$ $C)$ of penetrations passing through areas $\mathrm{V} 1, \mathrm{~V} 2$, and V3-V3A. For each penetration a representative field position was defined for each area, and it is shown with different symbols (dot, square, star) for each of the 3 regions. The representative positions of the fields in each region were obtained by averaging the horizontal and vertical coordinates of the centers of all response fields identified in that region. explored in the anterior bank of the lunate sulcus as a single region, the V3-V3A region, and have classified neurons to be located within its boundaries when the following criteria were met: anatomical reconstruction indicating as highly likely that the penetration had reached the anterior bank of the lunate sulcus; neurons were isolated at depths greater than $3 \mathrm{~mm}$ from the estimated surface of V1; identification during the penetration of 2 cellular regions as V1 and V2 and of 2 gaps (V1/V2, and below V2); neurons' response fields located several degrees away from the fields recorded in the regions above; response fields often large in size and difficult to define; and unpredictable shifts of receptive field location with small changes in electrode position or simultaneous recording from the same electrode of 2 neurons whose field centers were several degrees apart. Figure 4 illustrates the abrupt change in eccentricity observed in the "response field sequences" for 3 groups of penetrations traversing V1, V2, and V3-V3A. For each penetration 3 representative field positions are shown, marked with different symbols for each area.

The identification of the neurons' location was less certain for those few penetrations that passed through or very close to the fundus of the lunate sulcus, at or near the V2/V3 border. For some of these penetrations, the criterion listed above did not apply, and we cannot exclude that some neurons assigned to the V3-V3A region were actually located in area V2, or vice versa. On the other hand, the number of cells possibly classified incorrectly was small, and all had similar functional properties.

\section{Responses to correlated and uncorrelated binocular images}

A proportion of neurons in visual cortex, from 30 to $50 \%$ in different monkeys (Table 1), signal the state of binocular correlation for contrast texture by giving reciprocal responses, excitatory (activation) versus inhibitory (suppression), to the 2 opposite conditions. For any one neuron, the response to uncorrelation is usually the more evident and may be regarded as characteristic. Accordingly, we shall classify these "correlation neurons" as "activation neurons" or "suppression neurons" with respect to their response to uncorrelation.

Figure 5 illustrates the impulse activity of 2 correlation neurons in V1, one responding with activation, the other with suppression during binocular presentation (B) of uncorrelated stereopairs; the figure provides evidence of the binocular nature 
Figure 5. Replicas of impulse activity during left $(L)$ and right $(R)$ monocular viewing of a field of dynamic random dots, and binocular $(B)$ responses to dynamic random-dot correlograms for 2 neurons in area V1 (PH.31, MB.63). In this and following similar illustrations, each upstroke marks the occurrence of an impulse, and each horizontal sequence of impulses refers to a single behavioral fixation trial. Initially, the monkey looks at binocularly correlated (C) stereofields, and at $K$ it initiates the trial by pulling the reward key. After a delay of $500 \mathrm{msec}$ (time 0 ), the dot pattern within an area in the middle of the display becomes binocularly uncorrelated $(U)$ and then alternates with a correlated area for the period of steady eye fixation. In these examples, the left and right test fields were negatively correlated; their sizes are given to the right of the respective binocular record. For each stimulus configuration the activity recorded during several (4-8) successive behavioral trials is represented. The neurons whose records are illustrated above responded with activation to uncorrelation between left and right patterns, while its binocular activity was suppressed during presentation of correlated patterns. The neuron whose records are illustrated below responded with suppression to binocular uncorrelation and with binocular facilitation, or activation, to correlated images. The difference in impulse activity during monocular and binocular viewing is quite evident for both neurons.

\section{DYNAMIC RANDOM-DOT CORRELOGRAMS}

\section{ACTIVATION}

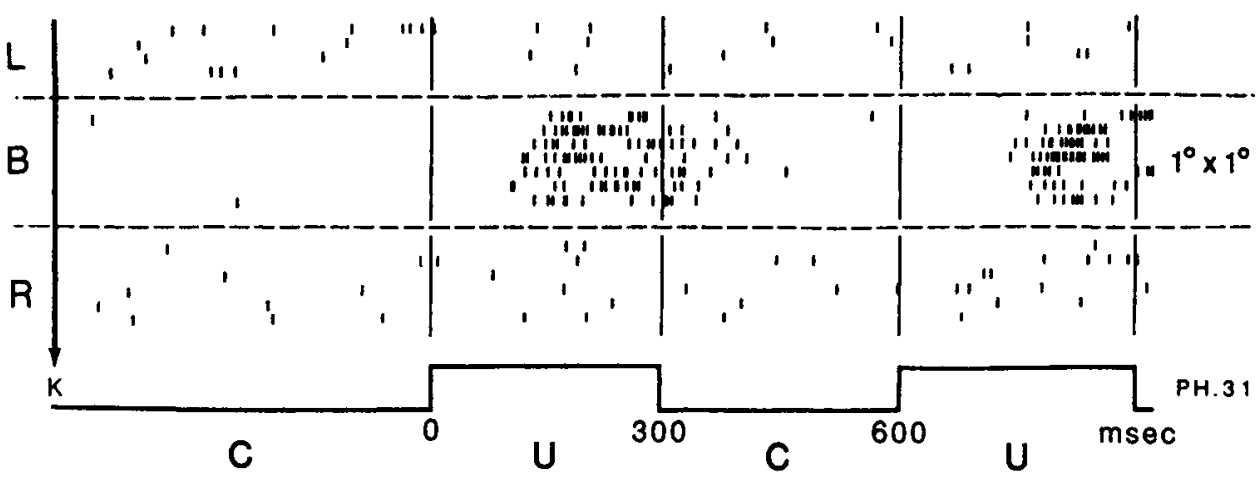

\section{SUPPRESSION}

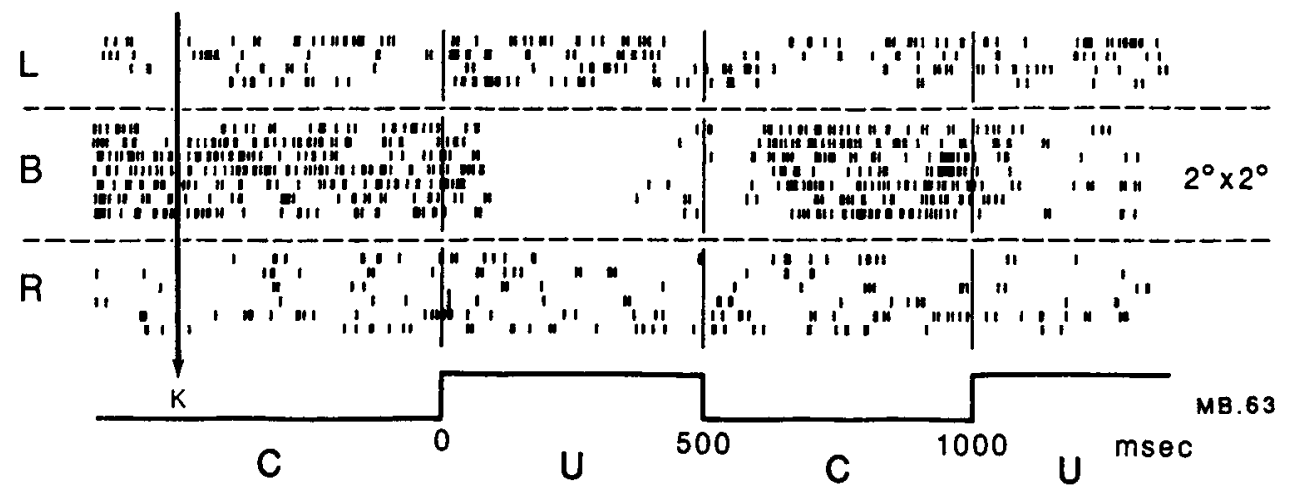

of these responses. As expected, left or right eye monocular display $(\mathrm{L}, \mathrm{R})$ of the random-dot field containing the respective half of the correlogram does not modify the ongoing neural activity. Correlated and uncorrelated binocular patterns, on the other hand, evoke obvious binocular responses. For the example shown in upper portion of Figure 5, uncorrelation (U) is associated with binocular facilitation, while correlation $(C)$ is associated with suppression. The lower example illustrates the opposite response behavior: binocular suppression to uncorrelation and activation to correlation.

The number of correlation-sensitive neurons was similar in $\mathrm{V} 1, \mathrm{~V} 2$, and $\mathrm{V} 3-\mathrm{V} 3 \mathrm{~A}$, with an average of $52 \%$ (Table 1; 2-monkey sample). The relative proportion of activation and suppression neurons varied considerably from one monkey to another, but activation neurons were more frequent than suppression neurons in all monkeys and in all areas. The activation:suppression ratio for $\mathrm{V} 1$ is $1.5: 1$ (114 neurons), and it is slightly higher for the smaller samples from V2 and V3-V3A. There appears to be no differential distribution of correlation-sensitive neurons in different cytoarchitectonic layers of area $\mathrm{V} 1$, and this is probably true of V2 and V3-V3A also.

The size of the field of uncorrelation and the number of uncorrelated pixels within the binocular receptive field were not important for evoking the effect, whether activation or suppression. For many neurons, correlation sensitivity was tested with patterns of different sizes, from the size of the minimum response field, as estimated with narrow bright bars, to arbitrarily large sizes. The response field associated with correlation was usually larger than the response field for contrast bars. In general, an area of uncorrelated texture the size of the bar has little or no effect, whereas areas several times that size evoke strong responses. The 2 examples $(A, B)$ in the upper part of Figure 6 (suppression) show that a neuron's response may be largely independent of the size of the field of uncorrelation, even though for some cells $(B)$ the minimum effective size may be relatively large. The rarely observed occurrence of an upper size limit was exhibited by the neuron of Figure 7 (activation) for which the best excitatory responses were obtained with uncorrelated stereopatterns $1^{\circ} \times 1^{\circ}$ in size, while larger areas were less effective.

Both activation and suppression are, in general, robust responses that are unaffected by pixel density or by the relative numbers of correlated and uncorrelated pixels. Impulse activity of essentially identical magnitude and temporal characteristics may be evoked, from any one neuron, by textures of different luminance and bright/dark pixel distributions (Figs. 6, 7). The differences between correlograms of different pixel density may be better appreciated by considering the 2 extreme conditions used in these experiments: at the lowest bright dot density (1\%), there is $1 \mathrm{dot} / \mathrm{deg}^{2}$ in the visual field of each eye for every display frame (15 msec); during the period of uncorrelation, the left and right single dots in the test area match, on average, only once every 100 frames. At the highest dot density $(50 \%)$, each eye sees $50 \mathrm{dot} / \mathrm{deg}^{2} /$ frame; during presentation of randomly uncorrelated fields, 25 left-eye dots and 25 right-eye dots are matched, whereas the other 25 in each eye are not. Under conditions of anticorrelation (Fig. 1), all dots in the test area are unmatched. 


\section{BRIGHT DOTS $20 \%$}

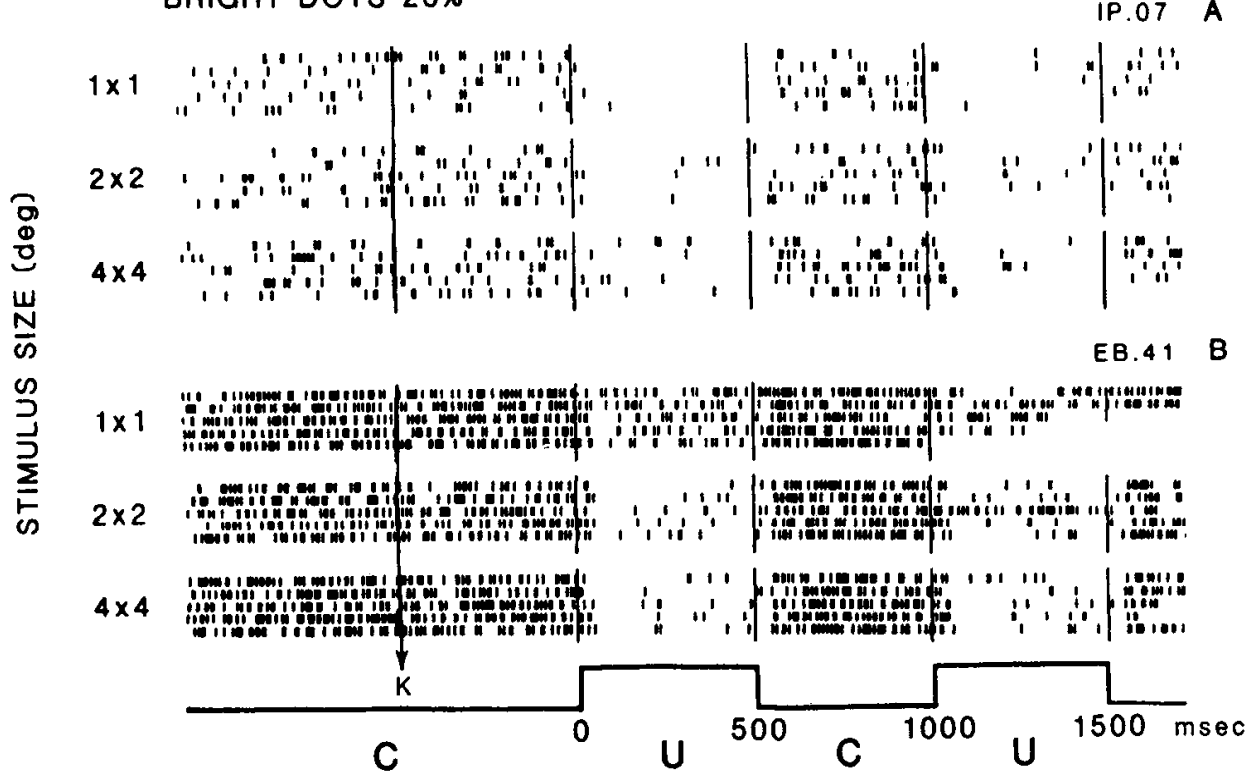

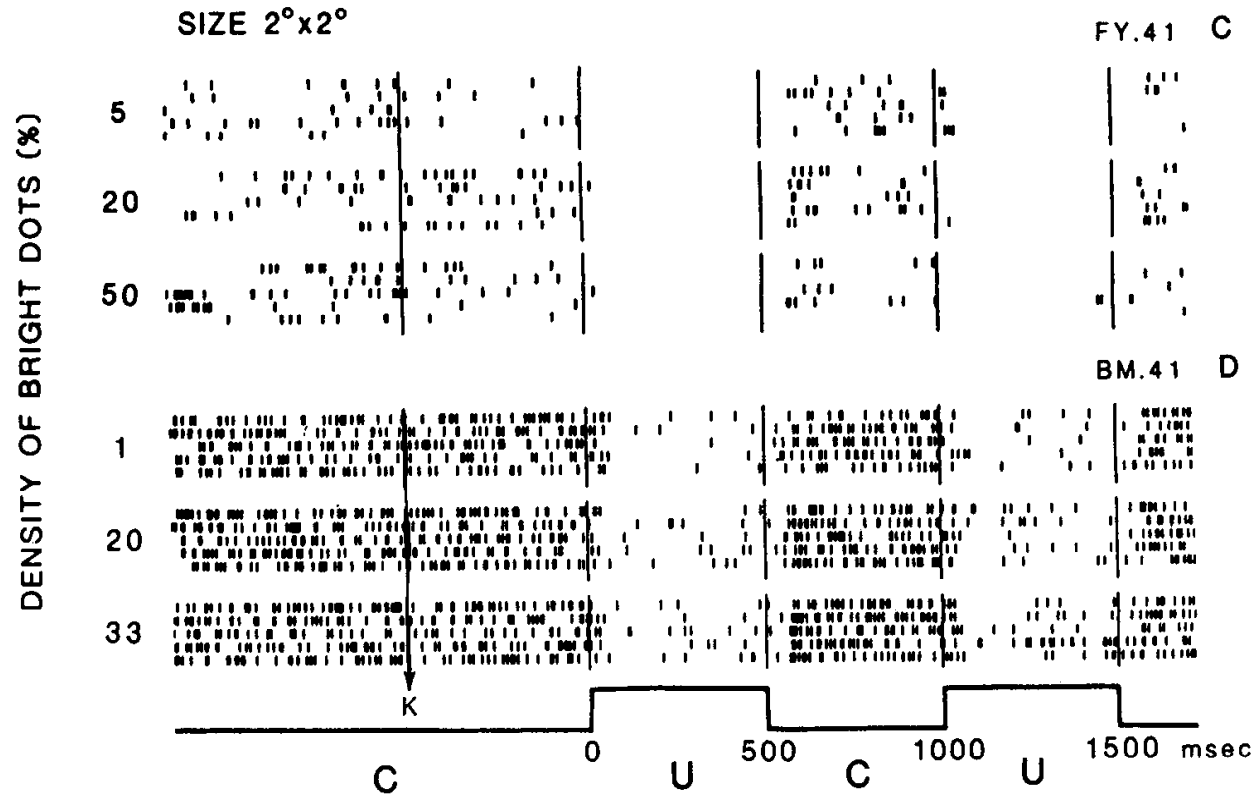

Figure 6. Suppression responses to binocular uncorrelation. Replicas of the impulse activity of 4 neurons during presentation of binocularly correlated $(C)$ and uncorrelated $(U)$ random-dot stereopatterns. These examples were selected to illustrate different degrees of activation by correlation. Three stimulus conditions are shown for each neuron, 5 replications for each condition. The neuron's response was largely independent of the extent of the area of uncorrelation and of the amount of mismatch. However, too small an areas was commonly ineffective. This was the case for all neurons when tested with areas of uncorrelation the size of their "optimal" contrast bar (given below). Indeed, for neuron $B$ even a $1^{\circ} \times 1^{\circ}$ stimulus evoked suppressive responses clearly smaller than those evoked by a $2^{\circ} \times 2^{\circ}$ or larger stimuli. Neuron $A$ was in $\mathrm{V} 1$, the other 3 neurons in V2. All neurons had a balanced ocularity and were orientation sensitive and directionally selective. Their receptive field centers were located between $1.2^{\circ}$ and $2.4^{\circ}$ from the vertical meridian. The optimal contrast bar size, orientation and speed of motion for each neuron were as follows: $A=1.1^{\circ} \times 0.2^{\circ}, 130^{\circ}, 4^{\circ} \%$ sec; $B=2.6^{\circ} \times 0.3^{\circ}, 25^{\circ}, 6^{\circ} / \mathrm{sec} ; C=$ $1.5^{\circ} \times 0.2^{\circ}, 125^{\circ}, 3^{\circ} / \mathrm{sec} ; D=2.2^{\circ} \times$ $0.2^{\circ}, 90^{\circ}, 4^{\circ} / \mathrm{sec}$.
Neurons tested for correlation sensitivity were analyzed separately for the coarse spatial arrangement of their receptive field, for ocular dominance, and orientation and direction selectivity. A classification as "complex" (C) and "simple" (S) was obtained for 208 neurons (V1 $=165, \mathrm{~V} 2=30, \mathrm{~V} 3-\mathrm{V} 3 \mathrm{~A}=13$ ) by comparing the spatial arrangement and characteristics of the binocular responses evoked by narrow bright and dark bars moving across the neuron's response field (Bishop et al., 1971; Schiller et al., 1976a; Kulikowski et al., 1981). The complex-to-simple ratio $(C: S)$ for the whole sample was similar to that reported by Schiller et al. (1976a), for area V1, about 1.5:1. Complex neurons, however, were more frequent than simple neurons among the correlation-sensitive cells; the C:S ratio was $7: 1$ for the activation neurons but only $1.6: 1$ for those responding with suppression. Correlation-insensitive neurons were equally distributed between simple and complex cells (Table 2). The ma- jority of correlation neurons gave responses of similar amplitude, or no response at all, to monocular stimulation of left and right eye with luminous bars. Ocular balance obtained for correlation neurons in V2 and V3-V3A, where it is typical of the general population (Baizer et al., 1977; Poggio and Fischer, 1977; Zeki, 1978a, b; Baizer, 1982; Burkhalter and Van Essen, 1986), but also in area V1, where ocular unbalance is more frequently found (IIubel and Wiesel, 1968; Schiller et al., 1976b; Poggio and Fischer, 1977; Poggio, 1984; Burkhalter and Van Essen, 1986). Similar observations were made for directional selectivity, correlation neurons being more frequently bidirectional than directionally selective in all 3 areas studied. The vast majority of neurons were orientation sensitive, although the tuning was usually rather broad (Poggio et al., 1977). None of these functional properties has any association with the binocular responses to disparity or correlation. 
BRIGHT DOTS $20 \%$

Figure 7. Activation responses to binocular uncorrelation. Replicas of impulse activity of a complex neuron (HN.07) in area V1, insensitive to positional disparity (flat). The optimal contrast bar was $0.6^{\circ} \times 0.1^{\circ}$ in size, orientation, $115^{\circ}$; speed of stimulus motion, $4 \%$ sec. Correlated patterns $(C)$ were shown during the period that includes the time before key down $(K)$ and between it and the presentation of the uncorrelated square ( $U$, at $t=0)$, a time during which the monkey was actively fixating the target. No excitatory effects are evident, and conditions of binocular correlation appear not to influence the activity of this cell, whereas uncorrelated figures evoke evident excitatory responses. The upper part of the figure shows that uncorrelated patterns evoke larger responses when relatively small in size $\left(1^{\circ} \times 1^{\circ}\right)$ than when larger, a rare occurrence for large uncorrelated images were generally as much or more effective than smaller ones. The lower part of the figure illustrates that the density of bright pixels was not an important parameter for determining the response amplitude or its characteristics.

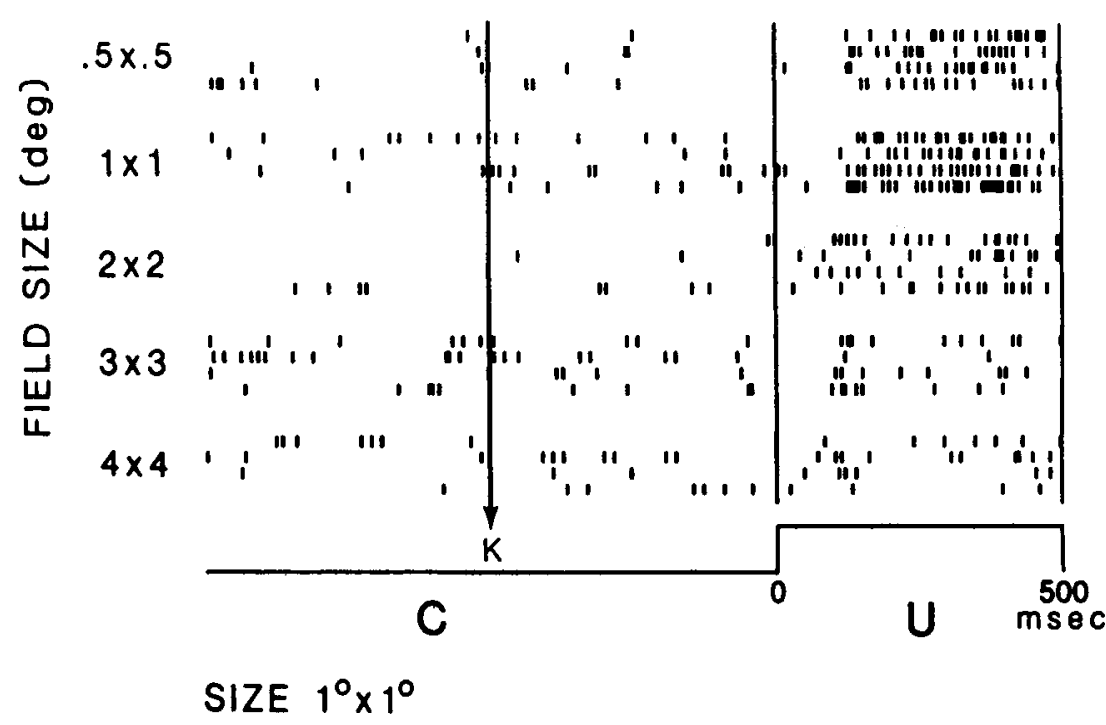

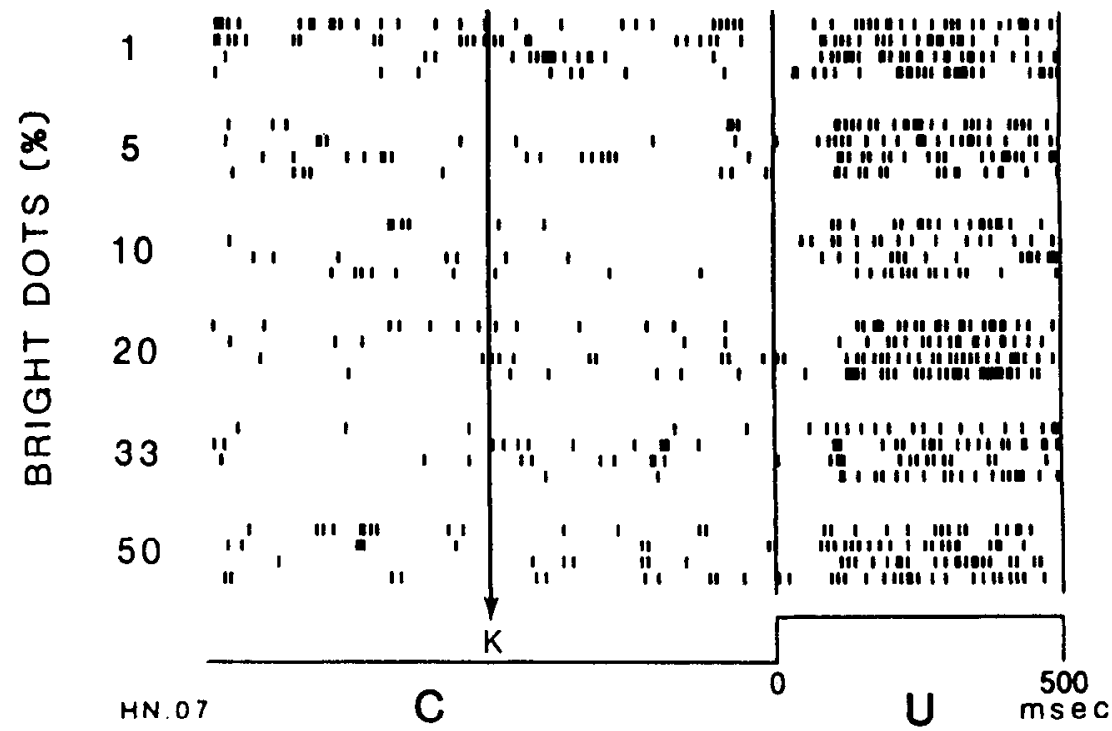

\section{Position-disparity selectivity}

A substantial proportion of neurons in the visual cortex of the macaque monkey give differential responses to horizontal binocular disparity. The amplitude and nature of the response varies along the disparity dimension and determines the disparity sensitivity profile of the cell (Poggio and Fischer, 1977; Poggio and Talbut, 1981; Maunsell and Van Essen, 1983; Poggio, 1984;

\begin{tabular}{|c|c|c|c|c|}
\hline \multirow[b]{2}{*}{$\begin{array}{l}\text { Response to } \\
\text { uncorrelation }\end{array}$} & \multicolumn{2}{|c|}{ Receptive field } & \multirow[b]{2}{*}{ Total } & \multirow[b]{2}{*}{$\begin{array}{l}\text { Ratio } \\
\mathrm{C}: \mathrm{S} \\
\end{array}$} \\
\hline & $\begin{array}{l}\text { Complex } \\
\text { (C) }\end{array}$ & $\begin{array}{l}\text { Simple } \\
\text { (S) }\end{array}$ & & \\
\hline Activation & 43 & 6 & 49 & 7.2 \\
\hline Suppression & 16 & 10 & 26 & 1.6 \\
\hline No response & 67 & 66 & 133 & 1.0 \\
\hline Total & 126 & 82 & 208 & \\
\hline
\end{tabular}

Poggio et al., 1985b; Burkhalter and Van Essen, 1986; Felleman and Van Essen, 1987; Hubel and Livingstone, 1987). Two major types of position-disparity selectivity have been recognized: tuned and reciprocal. The response profile of tuned neurons is characterized by binocular facilitation (tuned excitatory, TE) or binocular suppression (tuned inhibitory, $\mathrm{TI}$ ) over a narrow range of disparities, usually with opposite responses at one or both sides of maximum. The response profile of reciprocal neurons, on the other hand, is typically asymmetrical about the zero disparity, some cells giving excitatory responses over a more or less extensive range of crossed disparities and inhibitory responses over a similar range of uncrossed disparities (near neurons, $\mathrm{NE}$ ), other cells displaying the reverse response behavior, facilitation to uncrossed and suppression to crossed disparities (far neurons, FA). A small number of disparity-sensitive cells have response profiles that cannot be assigned with certainty to one of the above types (nonclassified, $\mathrm{nc}$ ). The term flat (FL) describes those neurons that give the same response at all disparities.

Figure 8 shows examples of the disparity-selectivity profiles 

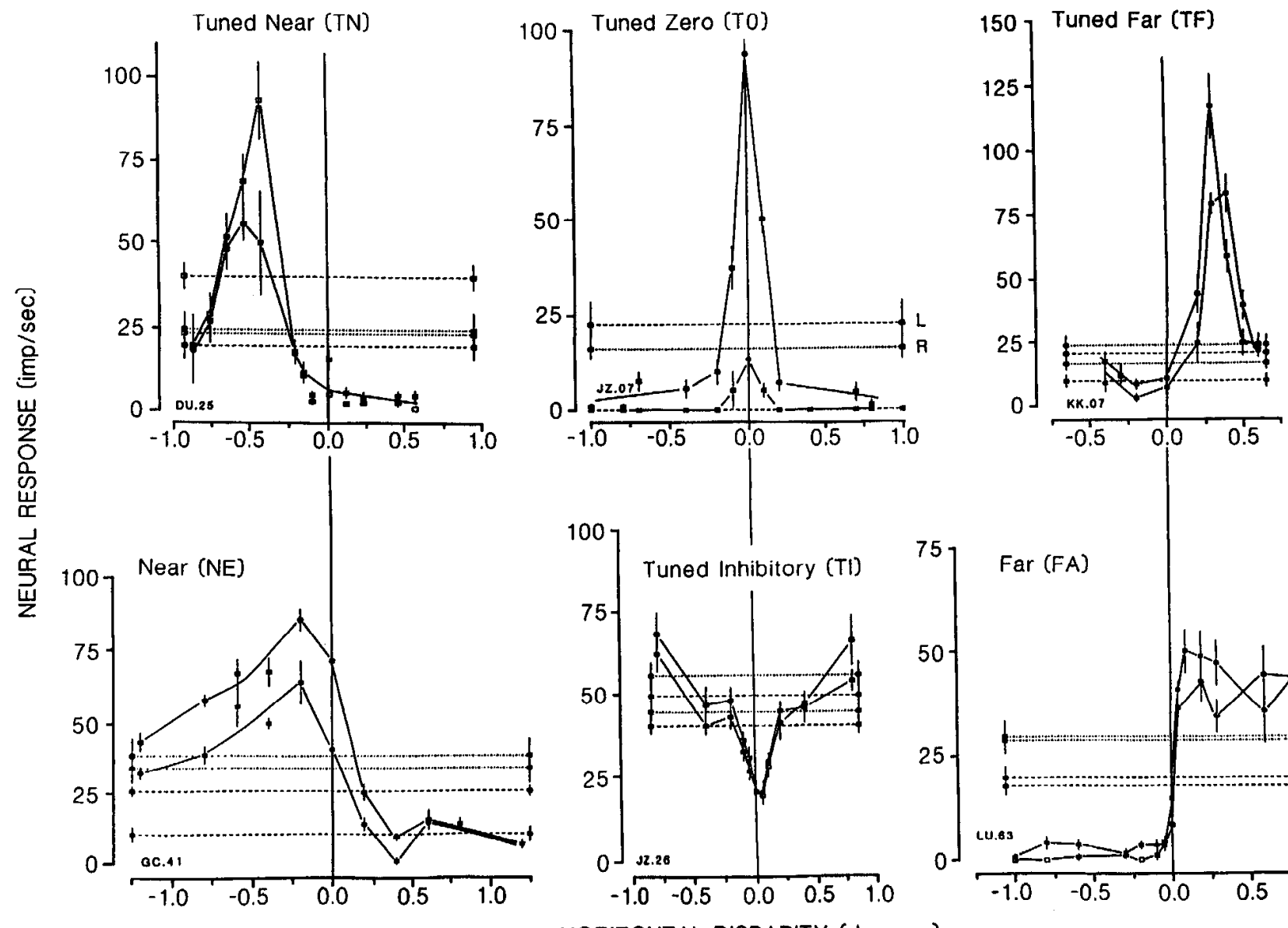

HORIZONTAL DISPARITY (deg arc)

Figure 8. Disparity sensitivity profiles of neurons in areas V1, V2, and V3-V3A of monkey visual cortex. Tuned zero (TO) excitatory neurons give maximal responses to binocular images of zero disparity. The tuned inhibitory $(T I)$ neurons are functionally reciprocal to the T0 neuron, their activity being suppressed rather than facilitated over a narrow range of horizontal disparities about and including zero disparity. The examples of the tuned far $(T F)$ and tuned near $(T N)$ both show maximal facilitatory responses at $0.4^{\circ}$ of disparity, uncrossed and crossed, respectively, and an inhibitory tail toward the zero disparity. Far $(F A)$ and near $(N E)$ neurons are characterized by a continuous, smooth, gradual transition from maximal excitation to maximal inhibition, with the midpoint of response activity along the disparity domain, at or very close to the zero disparity. All profiles were constructed by plotting mean response magnitude (impulses/sec) versus horizontal binocular disparity (deg arc) of a bright bar of "optimal" spatiotemporal configuration oscillating sideways across the neuron's receptive field. The bar's length ranged from $1^{\circ}$ to $3^{\circ}$, its width from $0.1^{\circ}$ and $0.3^{\circ}$. Rate of movement ranged between 2 and $8 \% \mathrm{sec}$. For each neuron, the response profiles to both directions of stimulus motion are shown, with the filled squares representing the responses to one direction and the open squares the responses to the diametrically opposite direction. Vertical bars indicate \pm 1 SEM. Lines are fitted to the data points by eye. The responses to monocular stimulation are shown by the horizonlul lines across the graph, identified at each end by the appropriate directional square symbols: broken line, left eye (L); dotted line, right eye $(R)$.

of cortical neurons that we have identified in areas in $\mathrm{V} 1, \mathrm{~V} 2$, and V3-V3A of the alert macaque. The characteristics of the various types have been described in some detail in previous publications (Poggio and Fischer, 1977; Poggio and Talbot, 1981; Poggio, 1984). The observations made in this study have confirmed our previous findings. In addition, they have revealed differences in the correlation sensitivity of TE neurons with different peak tuning (see below), suggesting the separation of these neurons in 2 categories: tuned excitatory neurons that respond maximally to binocular images with zero or non-zero disparity $\left( \pm 0.05^{\circ}\right)$, termed "tuned zero" (T0), and tuned neurons with peak response facilitation for horizontal retinal disparities greater than $0.05^{\circ}$, crossed or uncrossed, labeled "tuned near" (TN) and "tuned far" (TF), respectively. The 2 subtypes of tuned excitatory neurons differ in other functional characteristics in that TN/TF neurons have broader excitatory tuning curves than T0 neurons, usually with a clear inhibitory component tailing toward the zero disparity, and are less frequently directionally sensitive. Indeed, the stereoscopic response properties of TN/ TF neurons are similar to those of near/far cells, and it may well be that there exists a continuum of disparity selectivity profiles from the extended near/far profiles asymmetric about the zero disparity, to those with tuning peaks at disparities outside Panum's region. We shall present arguments later in this paper in support of the notion that the stereoscopic properties of the T0 and TN/TF types of excitatory neurons result from different forms of binocular interaction and suggesting that the TN/TF type depends on neural mechanisms similar to those generating the reciprocal near/far disparity selectivity profiles.

All types of disparity-selective neurons were found in all regions of cortex we explored. Estimates of the frequencies of occurrence of disparity-selective cells shows that binocular interaction controls and specifies the activity of the neuronal population in prestriate areas to a greater degree than in area V1. 

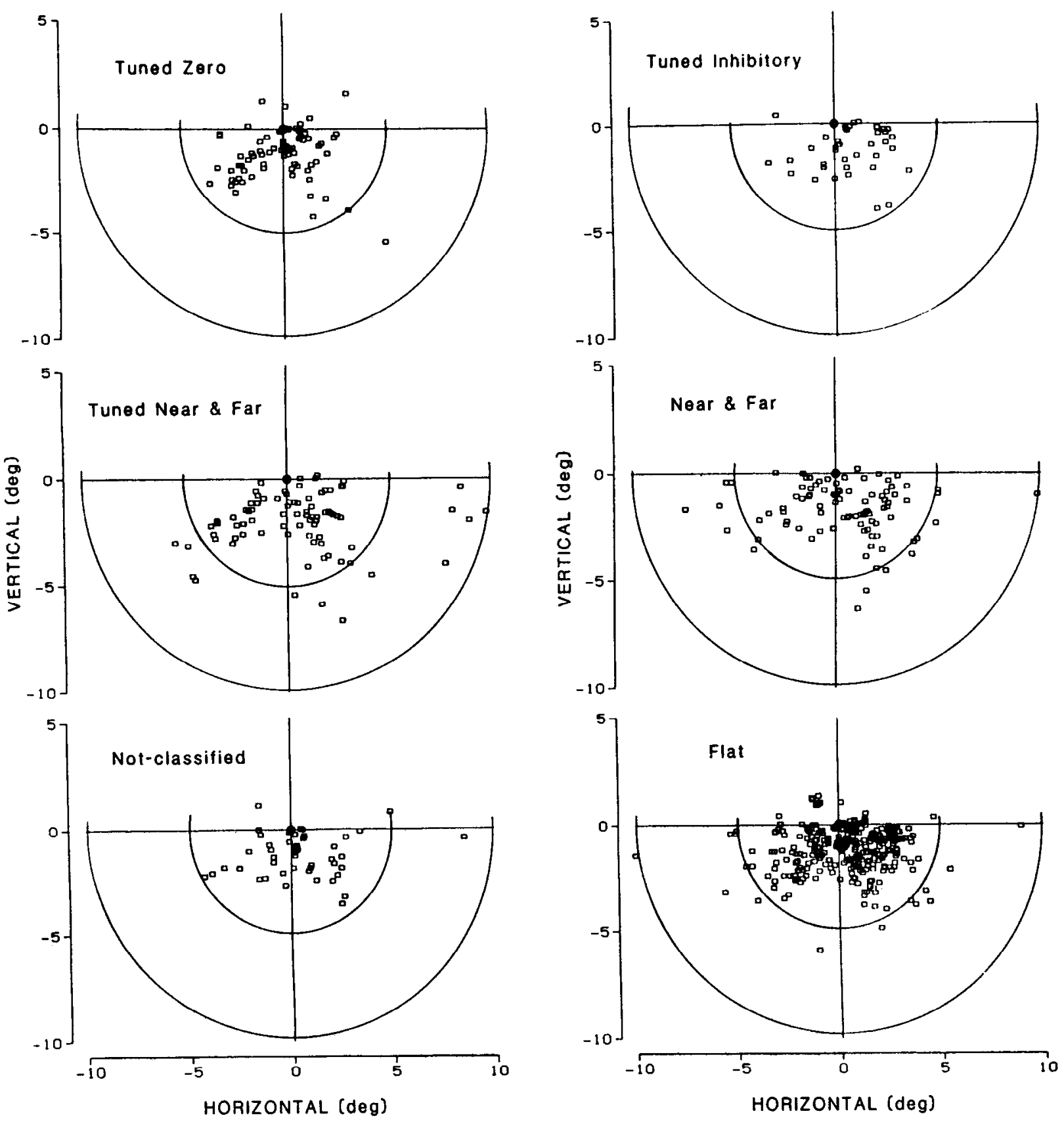

Figure 9. Plots of the locations of the receptive field centers for the 660 neurons of this study (7 monkeys) grouped with respect to their positional disparity sensitivity. The binocular depth properties of each neuron were determined with stereograms of narrow bright bars whose spatiotemporal configuration had been adjusted to evoke the best binocular response from the neuron.

The ratio of stereo to flat neurons in the 2-monkey sample was $1: 1$ for $V 1,2: 1$ for V2, and 4:1 for the V3-V3A cortex. These values, however, must be interpreted cautiously because of the small size of the sample and because of the bias introduced by the parcellation of neurons with different functional properties in different cortical areas (Van Essen and Zeki, 1978; Zeki, 1978b; Tootell et al., 1983; Livingston and Hubel, 1984; DeYoe and Van Essen, 1985; Shipp and Zeki, 1985; Van Essen et al., 1986; Hubel and Livingstone, 1987).

Disparity-selective neurons in area V1 are irregularly distributed, and although some types are found more often in some cytoarchitectonic layers than others (e.g., tuned excitatory, especially $\mathrm{T} 0$, in layers $4 \mathrm{~b}$ and 5-6) no clear-cut organization for depth has yet been identified in the striate cortex of the macaque (Poggio, 1984). Disparity-selective neurons in V2 were found segregated in zones extending through the depth of the cortex and interleaved with regions in which no stereo neurons were encountered. Similar observations were made by Hubel and Livingstone (1987), who found disparity-selective neurons in area V2 of the monkey in the cytochrome oxidase-stained "thick" stripes, and not in the "thin" or "pale" stripes. Neurons with stereoscopic properties in V3-V3A tend to occur in clusters. We have found numerous neurons with positional disparity selectivity in nearly all penetrations in the V3-V3A cortex totaling to $80 \%$ of the neurons studied in this region $(51 / 64)$. Felleman and Van Essen (1987) in the anesthetized macaque observed 


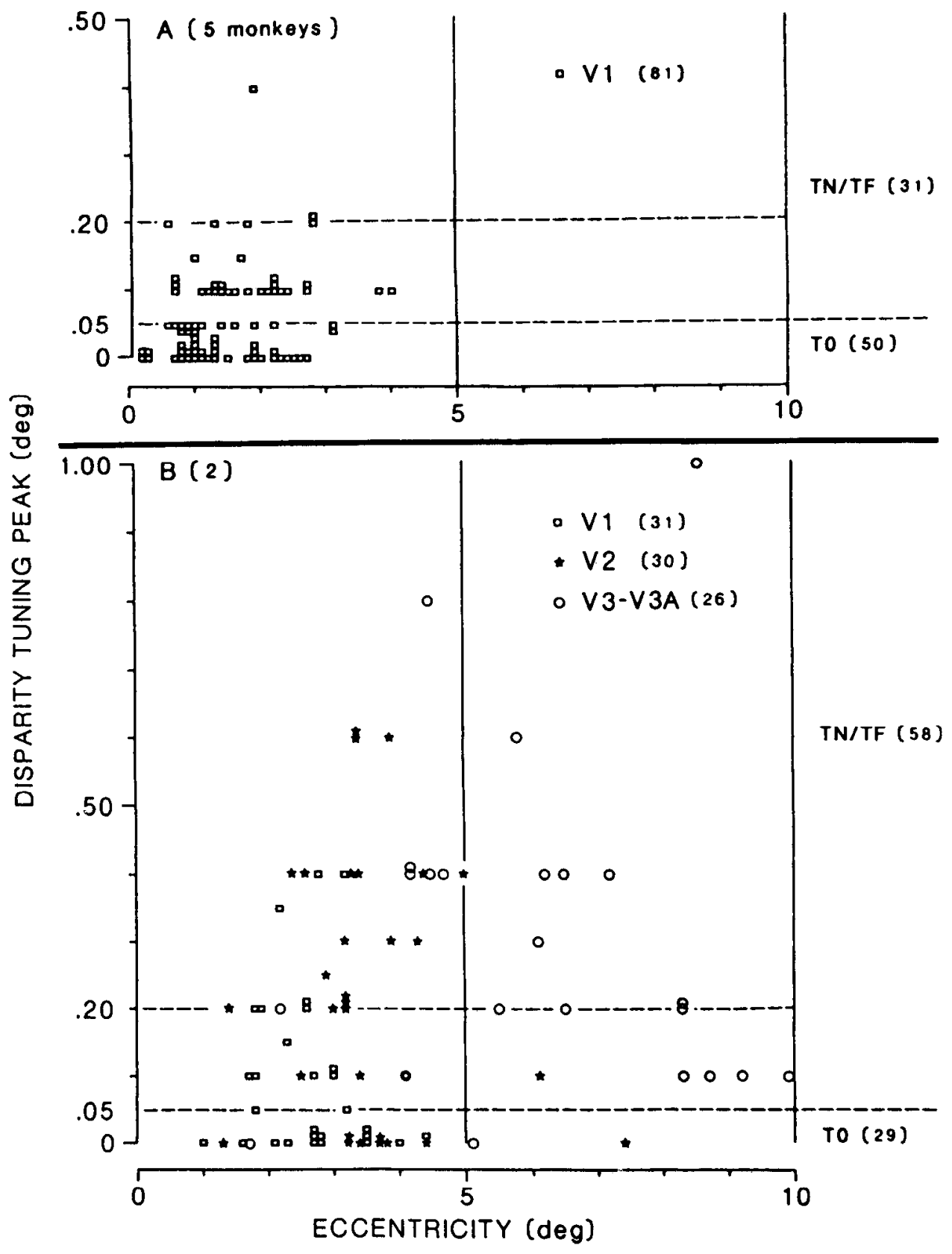

Figure 10. Plot of the disparities of maximal excitatory response of $\mathrm{TE}$ neurons versus the angular distance of their receptive field centers from the fixation target (eccentricity). The numbers in parentheses next to the labels for the disparity selectivity subtypes (TN/TF and $\mathrm{T} 0$ ) or the cortical regions ( $\mathrm{V} 1, \mathrm{~V} 2$, and V3-V3A) represent the number of neurons in that category. nearly half of the cells in V3 to be selective for positional disparity (20/46): About half of these cells were tuned excitatory at or near the zero disparity (similar to our $\mathrm{T} 0$ ), and the others had tuned inhibitory or asymmetric near/far profiles. We found tuned excitatory cells in V3-V3A in a similar proportion (26/ 51 ), but nearly all of them were TN/TF with peak excitatory responses at disparities away from zero, in the range between $0.1^{\circ}$ and $1.0^{\circ}$ (see Fig. 10).

The topographic distribution of the centers of the receptive fields of the neurons in the database of this study (660 neurons) is shown in Figure 9. All types of disparity-selective neurons, as well as flat neurons, are found at all eccentricities. A possible association between peak disparity tuning and ficld cccentricity was evaluated for the tuned excitatory neurons, and the results shown in Figure 10. In the 5 monkeys in which the central $4^{\circ}$ of visual field were explored in area V1, most TE neurons (62\%) were tuned to within $\pm 0.05^{\circ}$ of disparity and the rest (but one) at disparities around $\pm 0.1-0.2^{\circ}$ (Fig. 10 A). Stereo-tuned neurons with peak binocular facilitation at disparities larger than $0.05^{\circ}$, crossed or uncrossed, were isolated mainly in extrastriate areas. Figure $10 B$ shows the relation between tuning and eccentricity for 3 samples of similar size gathered in V1, V2, and V3-V3A. Tuned cells for larger disparities were observed at all eccentricities between $2^{\circ}$ and $10^{\circ}$. Neurons with peak tuning at disparities greater than $0.1^{\circ}$ were a minority in area V1 (14/31), increased in V2 $(21 / 30)$, and were common in V3 $(23 / 26)$. The sample from the V3-V3A region was of neurons with field eccentricity larger than $4^{\circ}$, whereas most of the neurons in $\mathrm{V} 1$ and $\mathrm{V} 2$ had more central receptive fields. These limited observations provide no evidence of a strict relation between eccentricity and stereotuning, but shows that in foveal striate cortex there exist a large population of neurons tuned for zero or near-zero disparities.

The general organization of the receptive fields of 140 dis- 


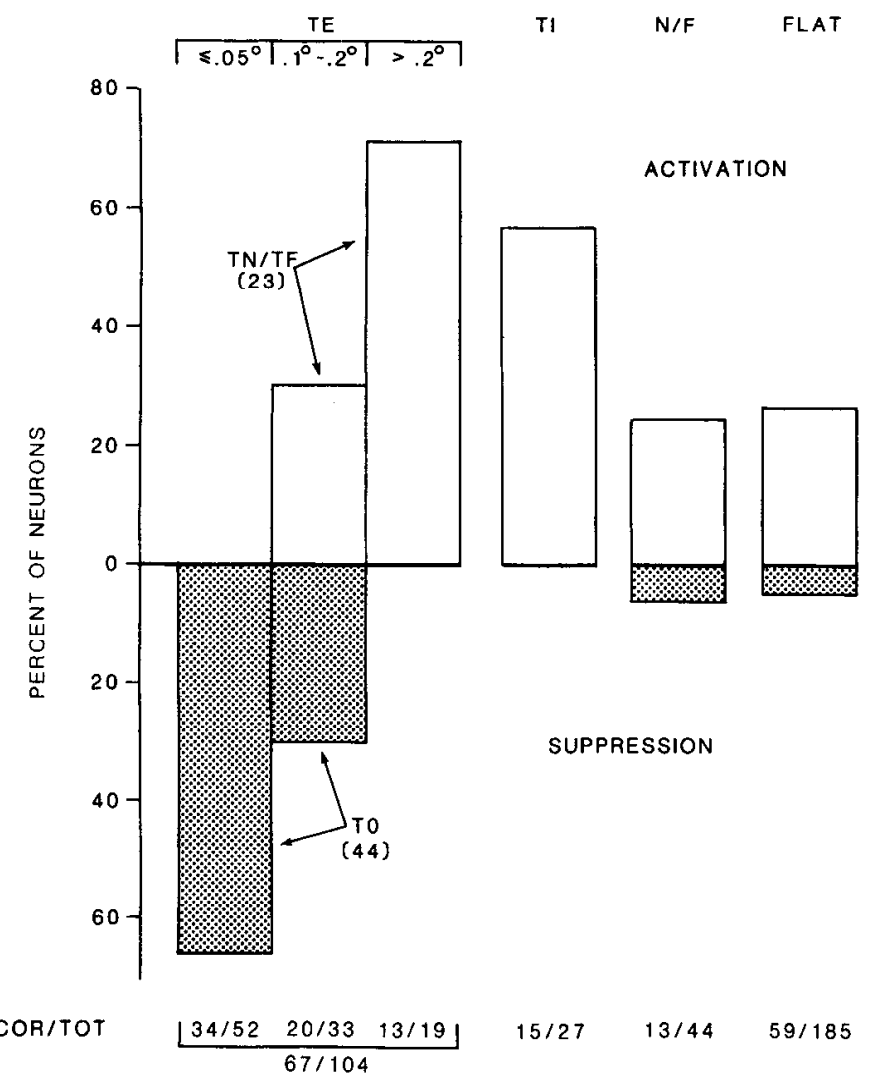

Figure 11. Histogram of the frequency of occurrence of suppression (stippled) and activation (clear) responses to image uncorrelation among neurons with different position-disparity selectivity. The labels above indicate the type of disparity profile. The numbers under TE show the disparity (in deg) at which the tuned excitatory ncurons gave maximal excitatory responses. The figures below the histogram indicate, for each disparity type, the number of correlation-sensitive cells $(C O R)$ and the total number of cells tested (TOT).

parity-selective neurons as "simple" or "complex" was derived by comparing the binocular responses evoked by moving narrow bars of opposite contrast, as described above. The distribution of disparity-sensitive types, both tuned and reciprocal were similar among simple cells (59) and complex cells (81), and we saw no evidence of any functional relation between simple/complex properties and disparity-sensitivity profiles for bar patterns. We have already indicated, though, this not to be the case for correlation-sensitive neurons, of which nearly $80 \%$ had complex receptive field propertics.

\section{Image correlation and positional disparity}

Correlation sensitivity may be part of the functional repertoire of disparity-selective neurons as well as of flat neurons. An analysis of the relation between disparity selectivity and image correlation sensitivity was conducted on a sample of 360 neurons studied quantitatively in V1 (277), V2 (51), and V3-V3A (32). These 2 neural properties are not independent of each other $\left(\chi^{2} p=0.0001\right)$ : The number of neurons that responded differentially to correlated and uncorrelated images and to positionally disparate stimuli, as well as the number of neurons that responded to neither, were significantly greater than the numbers predicted by chance association. Table 3 gives the distribution of the sensitive (yes) and insensitive (no) cells: $35 \%$ of the neurons lacked both properties; $38 \%$ of them displayed sen-
Table 3. Relationship between positional disparity sensitivity and correlation sensitivity for 360 neurons tested in V1, V2, and V3-V3A

\begin{tabular}{lllr}
\multirow{2}{*}{$\begin{array}{l}l \\
\text { correlation }\end{array}$} & \multicolumn{2}{l}{ Position disparity } & \\
\cline { 2 - 3 } Yes & No & $43 \%(154)$ \\
No & $27 \%(95)$ & $16 \%(59)$ & $57 \%(206)$ \\
& $22 \%(80)$ & $35 \%(126)$ & $100 \%(360)$ \\
\hline
\end{tabular}

sitivity for one type of stimulus and not for the other. The remaining neurons $(27 \%)$ signaled correlation as well as disparity; the vast majority of these neurons were disparity-tuned neurons (82/95), especially tuned excitatory.

We have observed a consistent relation among the tuned excitatory neurons between peak tuning and response to uncorrelation. The nature and extent of this relation are illustrated by the histograms of Figure 11. Of the correlation-sensitive neurons, all those with disparity tuning within $\pm 0.05^{\circ}$ responded to uncorrelation with suppression; all neurons with disparity tuning at $\pm 0.20^{\circ}$ or larger responded with activation. Neurons with peak disparity about $\pm 0.10^{\circ}-0.20^{\circ}$ split in their response behavior: half respond to uncorrelation with suppression and the other half with excitation. On the assumption that the peak tuning value assigned to these neurons may reflect experimental and natural variability, those neurons whose activity was suppressed by uncorrelation were classified as tuned zero (T0) neurons and those that were activated as tuned near or tuned far (TN/TF), depending on whether they responded to crossed or uncrossed disparities. The response of the tuned inhibitory neurons, whose peak tuning was nearly always at zero or near-zero disparity, was predictably opposite that of the tuned zero excitatory neuron; of the TI neurons, $56 \%$ responded to uncorrelation, all with activation. Correlation sensitivity was less frequently observed among the reciprocal stereo neurons. About a third of the cells with near/far disparity profile responded to uncorrelated images, nearly all with activation (11/13). Correlation sensitivity was also exhibited by a third of the disparityinsensitive, FL neurons; for these also, activation responses were observed 5 times more frequently than suppression responses.

Figure 12 (left) illustrates the typical responses of a T0 neuron to solid bar stereograms. The range of binocular facilitation is narrow $\left( \pm 0.1^{\circ}\right)$ with peak at zero disparity. Stimuli of larger disparities, either crossed or uncrossed, have inhibitory effects. The plot at lower right shows the disparity sensitivity profile for this cell. In the inset in upper right is the response of this neuron to stereofields of dynamic random dots: The neuron's activity is maintained at a high level as long as the dot patterns are the same in the 2 eyes, but it is completely suppressed when the patterns are binocularly uncorrelated.

The relation between the sensitivity to positional disparity and to contrast texture correlation for single neurons in the visual cortex of the alert monkey is illustrated in Figure 13. It shows the responses of 4 neurons to bar stereograms (left panel) and to dynamic random-dot correlograms (right panel). At the top of the figure is an example of a neuron tuned to zero disparity (T0) whose activity is suppressed by uncorrelation. It is shown here to allow direct comparison with the other neurons illustrated: a tuned excitatory with peak responses at $-0.4^{\circ}(\mathrm{TN})$, a tuned inhibitory neuron (TI), and a flat neuron (FL) that responded over a large range of disparities. These 3 neurons, like 

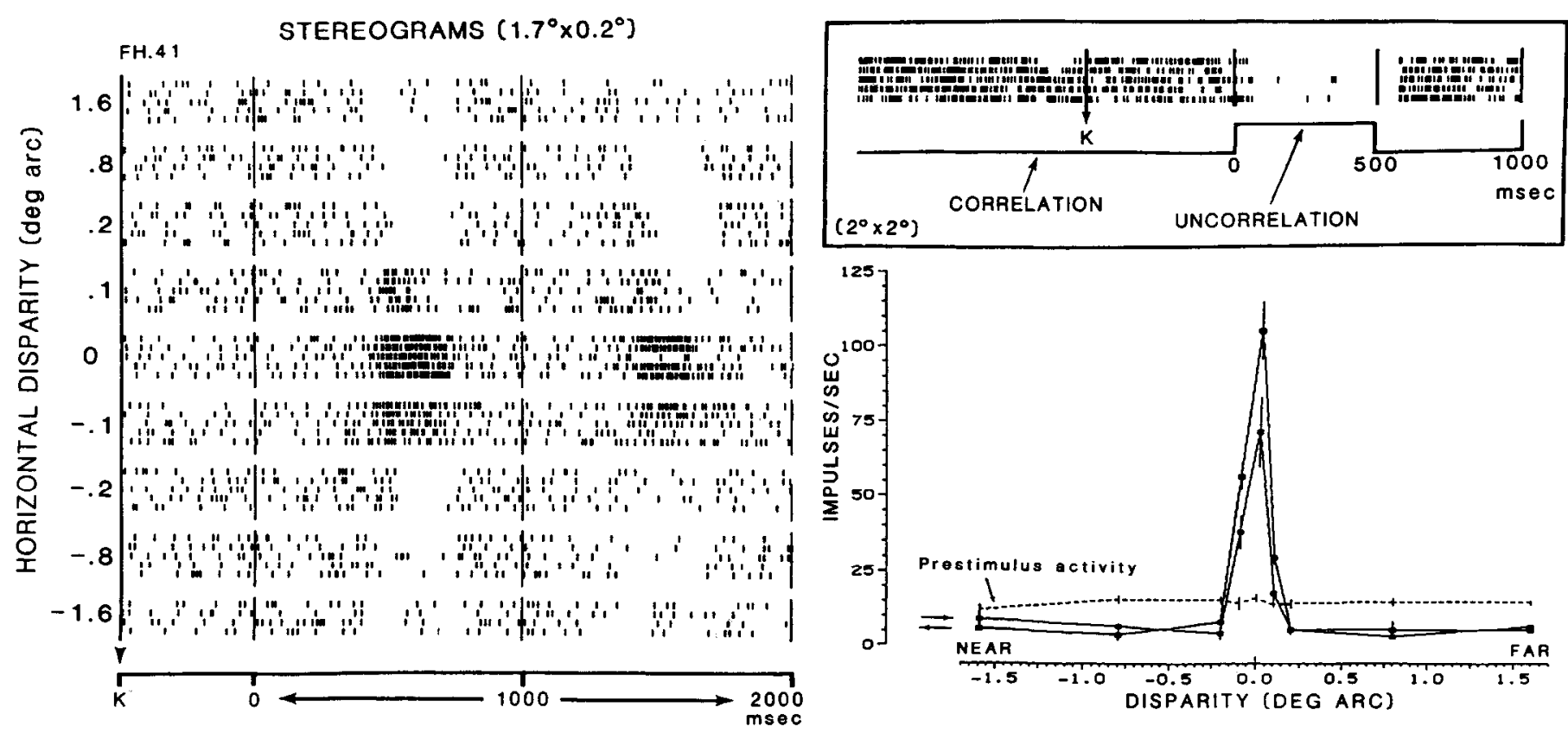

Figure 12. Tuned excitatory neuron in area V1 with peak response at zero disparity (FH.41). The panel at left shows a set of responses to stimuli of different horizontal disparity, both crossed (-) and uncrossed. The stimulus was a luminous bar (100\% dot density) appearing on a dark background and moving bidirectionally at $6^{\circ} / \mathrm{sec}$ across the receptive field (bar size $=1.7^{\circ} \times 0.2^{\circ}$; orientation, $35^{\circ}$ from vertical). As commonly observed, the variability between responses at a given disparity was consistently smaller than the difference between responses at different disparities. The disparity sensitivity profile (lower right) was constructed by plotting the average impulse frequency occuring within a time window ( 300 msec) centered on the discharge peak; vertical bars indicate \pm 1 SEM. Insert (upper right), Response of this neuron to dynamic random-dot correlograms. Test area was $2^{\circ} \times 2^{\circ}$, bright dot density $20 \%$. Thus, left and right test squares contained, on average, $32 \%$ uncorrelated (bright/dark) dot pairs, $4 \%$ bright dot pairs, and $64 \%$ dark pairs. Mean luminance of the display was $2.7 \mathrm{~cd} / \mathrm{m}^{2}$.

all of their classes, respond to uncorrelation with activation, in contrast to the T0 neuron.

It is not clear why all neurons that respond to solid bar stereograms do not also respond to random-dot correlograms. Indeed, a substantial proportion of neurons in our sample gave differential responses to only 1 of the 2 kinds of stereo stimulation and not to the other $(139 / 234$, or $60 \%)$, with only a minority of $40 \%$ of cells responding to both (Table 3 ). A larger selection of stimuli to test the neuron's stereo properties (short line segments of various orientation; dots or other simple figures of different size and/or contrast, etc.) may provide explanatory information.

\section{Discussion}

\section{Operational properties of binocular neurons}

The results of these experiments show that 2 aspects of the visual images associated with binocular vision, relative horizontal position and contrast correlation, interact in determining the binocular responses of cortical visual neurons. On the basis of their stereoscopic response properties, 3 major operational groups of neurons may be recognized in all areas of cortex that we have explored, V1, V2, and V3-V3A.

\section{Stereo-tuned neurons with peak responses within Panum's region $(T 0, T I)$}

(1) A majority (67/104) of the tuned excitatory neurons responded to dynamic random-dot correlograms. Of these, all neurons with disparity tuning peak within \pm 3 min arc (tuned zero neurons, T0) and half of the tuned-near and tuned-far neurons (TN/TF) with peak binocular facilitation about $\pm 6-12$ min arc, were characteristically suppressed by uncorrelated pat- terns. These neurons therefore give binocular facilitory responses to objects located in their field of view at or about the horopter and are silenced, in the absence of such objects, by the images of different objects at other depths. Earlier findings in area V1 of the anesthetized cat-such as the suppression ("veto") of the binocular response by images at disparities larger than optimal described by Barlow et al. (1967) or the inhibitory interaction reported by Bishop et al. (1971) with image misalignment-may be the same phenomenon. (2) Half $(15 / 27)$ of the neurons with disparity selectivity profiles opposite to that of the T0 neurons (tuned inhibitory neurons, TI) responded to dynamic random-dot patterns. All these neurons were suppressed by binocular texture correlation and activated by uncorrelation.

These tuned excitatory and tuned inhibitory neurons that respond over a narrow disparity range centered at or near zero disparity may be regarded as contributing in a complementary way in the neural definition of the horopter and of Panum's fusional area. The T0 neurons are activated and the TI neurons are suppressed by objects about the fixation distance and, reversing their response, respectively inhibited and excited by nearer or farther objects. The ongoing activity of these 2 functional sets of neurons, the discharging and the silent ones, may provide the basic substrate for fine stereopsis (Bishop and Henry, 1971; Poggio and Fischer, 1977) and may represent the essential mechanisms for binocular single vision, carrying information for fusional mechanisms, both sensory and motor.

\section{Stereo-tuned neurons at larger disparities and neurons with} reciprocal disparity selectivity (TN/TF, NE/FA)

A subset of the tuned excitatory neurons with peak binocular facilitation at disparities at about $\pm 6-12 \mathrm{~min}$ arc, and all those 

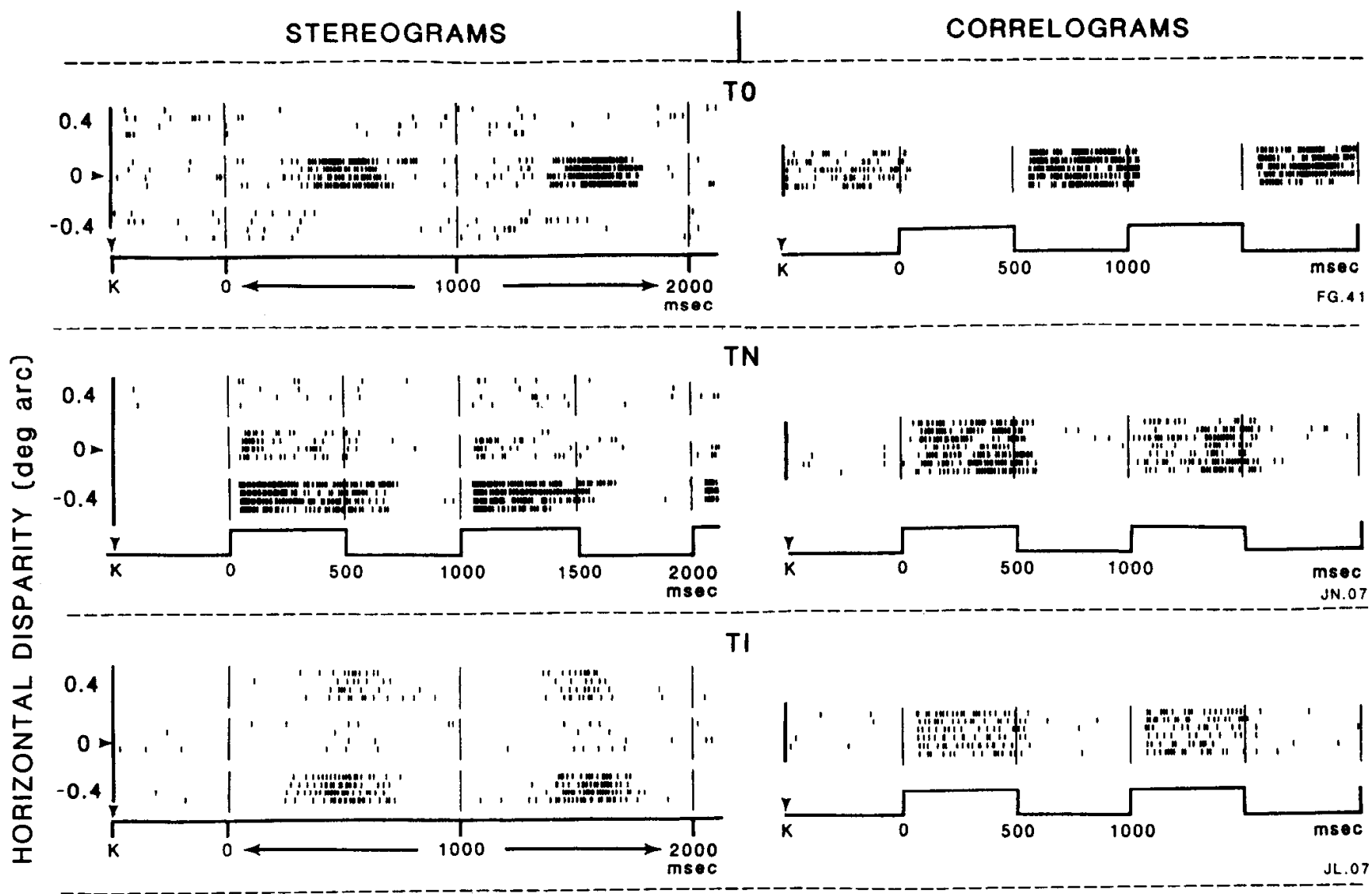

FL

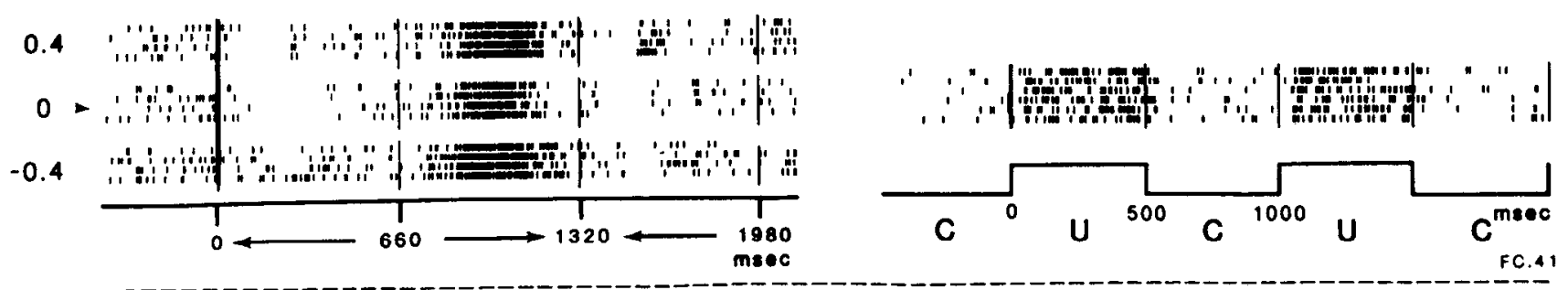

Figure 13. Replicas of responses of 4 cortical neurons to binocular disparity (stereograms) and binocular correlation (correlograms). Left, Three sets of responses for each neuron to stereograms of different disparities: $0.4^{\circ}$ crossed $(-), 0.4^{\circ}$ uncrossed, and $0^{\circ}$. The stereograms were constructed with bars of intensified dots (luminance $=14.4 \mathrm{~cd} / \mathrm{m}^{2}$ ) adjusted in spatiotemporal configuration, and they appeared against a background chosen for optimal binocular responses: a dark background for top and bottom examples or a background of dynamic random noise (mean luminance 2.7 $\mathrm{cd} / \mathrm{m}^{2}$ ) for the 2 middle examples. The 4 neurons had different stereoscopic selectivities: $T 0$, tuned excitatory at zero disparity (area V1); $T N$, tuned excitatory near, peak response at $-0.4^{\circ}$ of disparity (V2); $T I$, tuned inhibitory with peak at $0^{\circ}$ of disparity (V1); $F L$, disparity-insensitive neuron, unidirectional (V1). Right, Impulse activity in response to presentation of random-dot stereopairs alternating between image correlation $(C)$ and image uncorrelation $(U)$. The T0 neuron is suppressed by binocularly uncorrelated dot patterns and strongly activated by correlated ones. All other neurons respond in the opposite fashion: activation by uncorrelation and no excitatory response (suppression?) to correlation. The correlograms were constructed with square areas (size $2^{\circ} \times 2^{\circ}$; dot density, $50 \%$; mean luminance, $7.0 \mathrm{~cd} / \mathrm{m}^{2}$ ) centered on the neuron's receptive fields and surrounded by a correlated background of the same density.

with tuning at disparities larger than \pm 12 min arc, responded to binocularly uncorrelated images with activation and never with suppression. A similar activation by uncorrelation was observed in about $30 \%$ of the neurons with extended, reciprocal disparity profiles. For all these TN/TF and NE/FA neurons, however, the responses to uncorrelated images were usually smaller, and never larger, than the responses evoked by correlated images at the optimal excitatory disparity. This may indicate that these responses are the responses to those elements of the uncorrelated stereopair that occur with the correct excitatory disparity for the neuron. It may be that the depth response profile of all these cells is determined chiefly by positional disparity sensitivity, with image contrast correlation playing a less specific role in the stereo selectivity of these cells. Whether the asymmetrical inhibitory component of the response profile that is characteristic of NE/FA neurons, and is often present for TN/ TF neurons (Fig. 8), is evoked by disparities opposite in direction to those evoking excitation remains to be investigated. Under conditions of normal binocular vision, these neurons would maintain a certain level of activity in the absence of stimuli within their "stereoscopic response field" (Poggio and Talbot, 1981) and will respond with an increase or decrease of 
ACtIVATION

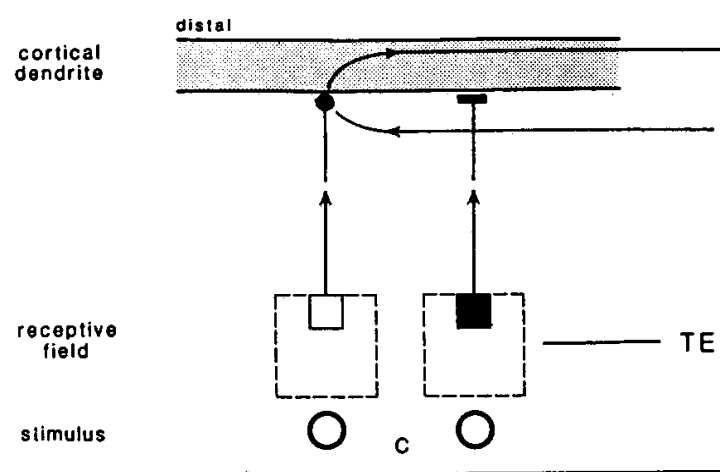

SUPPRESSION

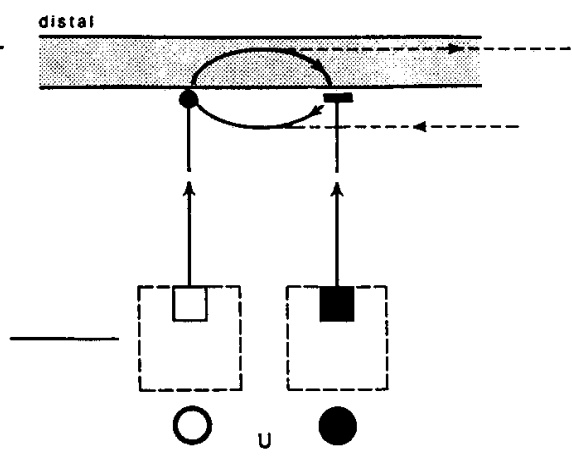

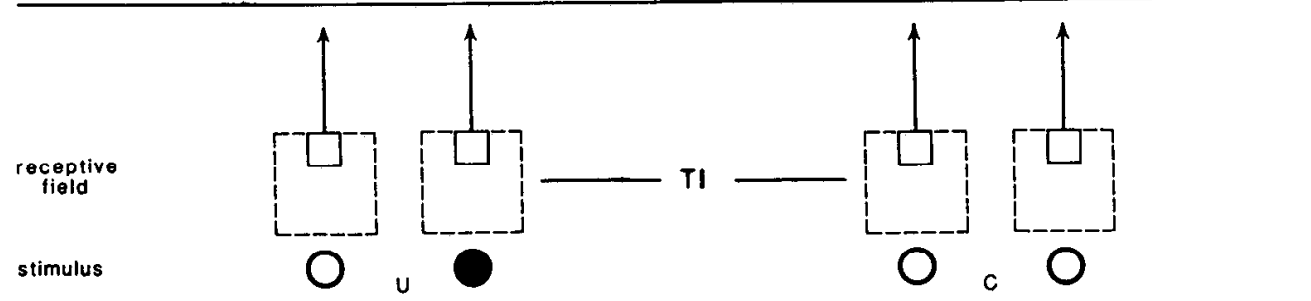

Figure 14. Schematic representation of hypothcsized interaction of binocu lar inputs at nearby synapses on the cortical dendrite of stereo neurons sharply tuned to zero or near-zero disparities. Tuned excitatory $(T E)$ neurons respond with activation when one eye signals bright while the other eye does not signal dark from the corresponding subfield. Tuned inhibitory $(T I)$ neurons are continuously active except when both eyes signal bright or dark. Clear area in receptive field outline indicates subfield sensitivity to bright, and black area to dark. Clear and black circles indicate bright and dark stimuli. $C$, correlation; $U$, uncorrelation (see text). activity to objects of appropriate configuration located at different depths within their space of receptive field binocular interaction. These populations of TN/TF and NE/FA neurons may operate in the neural processing leading to coarse stereopsis (Bishop and Henry, 1971; Poggio and Fischer, 1977), as well as part of the system controlling binocular vergence. Moreover, the near/far neurons could effectively contribute to or indeed determine stereoacuity by signaling small disparity changes through their finely graded response transition from suppression to facilitation about the fixation distance (Poggio, 1984).

\section{Disparity-insensitive neurons ( $F L)$}

About 25\% of the "flat" neurons that were tested for correlation sensitivity responded to uncorrelation with an increase of their impulse activity. FL cells were only rarely (5\%) suppressed by uncorrelated visual patterns. The functional role of the FL cells in stereopsis, if any, is not understood.

\section{Neural mechanisms of stereopsis}

The experimental evidence provided by this and other sludies suggests that the stereoscopic properties of binocular neurons in the visual cortex depends upon an orderly arrangement of the inputs from left and right eyes to the cortical cell, an arrangement that provides for a precise binocular matching of the receptive fields' subregions, whether spatially in register or disparate. This matching must be set up at the earliest stages of binocular convergence, and it is likely to be supported by the spatial distribution of the geniculate terminals in the striate cortex. Blasdel and Fitzpatrick (1984) have shown that throughout layer IVc of the macaque there exists a finely scaled and orderly retinotopic map of nonoriented receptive fields both for the magnocellular (IVc $\alpha$ ) and the parvicellular $(\operatorname{IVc} \beta)$ inputs. This organization could indeed provide for the precise binocular registration of the receptive fields of neurons in superficial and deeper layers of area V1.
At any one time, the impulse activity of cortical neurons reflects the interaction of the binocular inputs set up by the relations between retinal image features and functional properties and spatial arrangement of the receptive field subregions. For any one neuron, different matches along the position disparity domain evoke a series of responses (the neuron's disparity selectivity profile) as determined by the number, position, size, and functional properties of the subregions in the receptive fields (Ferster, 1981; Poggio and Talbot, 1981; Poggio and Poggio, 1984; Maske et al., 1986a, b).

The results of experiments on paralyzed/anesthetized cats (Barlow et al., 1967; Nikara et al., 1968; Henry et al., 1969; Joshua and Bishop, 1970; Bishop et al., 1971; Ferster, 1981) suggest that the positional disparity selectivity of cortical neurons is based on a slight relative displacement of the neuron's receptive fields in the 2 eyes (receptive field disparity). Alternatively, it could be that the 2 monocular fields are in topographic register but with their subfields binocularly "wired" for disparity, though, for any one neuron, not necessarily all for the same disparity. Dow et al. (1981) have bcen ablc to define the extent of the binocular response field of cortical neurons in the alert monkey in a reproducible manner to within a few minutes of arc. In our experience, however, manual mapping has given results that were sufficiently variable to blur possible small differences between the outlines of the 2 monocular fields and between them and the binocular field. We were unable, therefore, to establish directly receptive field disparity, or its nature. It may be assumed, however, that for disparity tuned cells, whether excitatory or inhibitory, the "optimal" match obtains when retinal images and receptive fields are in spatial register, and these conditions of maximal binocular correlation evoke maximal binocular responses (Bishop et al., 1971; Maske et al., $1986 \mathrm{a}, \mathrm{b})$. For the near/far stereoscopic neurons, on the other hand, we do not know which of the responses along the disparity domain is associated with the exact registration of fields and 
images, nor do we know whether the receptive fields of these neurons in the 2 eyes are positionally corresponding or disparate. Ferster (1981) interpreted the disparity selectivity profile of near/far neurons in the anesthetized and paralyzed cat as due to an opponent ocular unbalance between the inputs from the 2 eyes, originating from positionally disparate response field, one excitatory and the other inhibitory. The spatial arrangement of the receptive fields of disparity-selective neurons in the alert macaque is yet to be determined.

Our observations show that similar proportions of simple and complex neurons are sensitive to the binocular disparity of narrow contrast bars. We have obtained no evidence of any association between simple/complex properties and disparity sensitivity profiles. The receptive fields of simple cells are composed of adjacent and overlapping On and Off subregions that can be shown directly with stationary flashing bars or revealed with moving edges that evoke a series of response peaks in a one-toone correspondence with the subregions. In the striate area, simple fields are usually composed of 2-4 subregions (Hubel and Wiesel, 1962, 1968; Schiller et al., 1976a; Mullikin et al., 1984a; Camarda et al., 1985a, b; Maske et al., 1985), but cells have been described whose fields include up to 6 excitatory regions alternating in space with up to 7 inhibitory regions (Mullikin ct al., 1984b). The structure of the receptive fields of complex cells is more difficult to determine because these fields do not have directly recognizable On and Off subregions. The notion that the fields of complex cell also were composed of subunits was first advanced by Hubel and Wiesel (1962), who hypothesized that complex cells receive their input from a series of spatially adjacent simple cells. Subfields in complex cells have been identified on the basis of the responses obtained with sinusoidal gratings of different spatial frequency (Movshon et al., 1978; Glezer et al., 1980) or random-luminance bar patterns (Emerson et al., 1987).

There is suggestive evidence that an even finer binocular "wiring" may exist as shown by the disparity selectivity of a many cortical neurons for dynamic random-dot stereograms despite the abundant ambiguous matches between dots that are possible in these stereopatterns (Julesz, 1960, 1971; Poggio, 1980, 1984; Poggio et al., 1985a). The size and number of the subregions of the simple receptive fields are likely to provide too coarse a binocular pairing for supporting a mechanisms for the detection of the correct disparity in random-dot stereograms. Indeed, nearly all neurons responding to random-dot stereograms are complex neurons (Poggio, 1984; Poggio et al., 1985b) whose receptive field structure is more detailed than that of the simple cells. This could result, for example, by the convergence of inputs from simple cells, as in the hierarchical model of cortical organization of Hubel and Wiesel (1962), but other arrangements making up a "complex" field are clearly possible; moreover, this arrangement need not be, for any one neuron, unique and fixed. Poggio and Poggio (1984) have suggested a schema of binocular matching that is based on a disparity organization that includes sets of discrete and numerous receptive sites in the receptive field of one eye "gated" with a similar set of appropriate positional disparity in the other eye. The size of the subfields should be of the order of the size of the associated monocular receptive fields at the cortical input (layer IVc).

On these considerations, models of neural organization may be suggested to account for the experimentally observed disparity selectivity. They are based on known aspects of synaptic interaction: linear summation between distant excitatory and inhibitory synapses followed by a threshold (and gates) and nonlinear shunting inhibition of proximal synapses upon excitation at nearby distal synapses (AND-NOT gates) (Rall, 1964; Koch et al., 1983). These types of models assume that the inputs from the 2 eyes in area V1 are in a precise and detailed retinotopic register; that spatially matched subtields in left and right eyes signal either opposite luminous contrasts, bright (On) and dark (Off), or the same contrast (bright or dark for both eyes), and that these signals act in parallel at numerous independent synaptic subunits throughout the neuron's dendritic tree.

\section{TO and TI neurons}

Tuned excitatory and tuned inhibitory neurons whose binocular inputs are matched for zero or near-zero disparities may derive their stereoscopic properties from a shunting type (AND-NOT) of synaptic interaction (Fig. 14). A model based on such a mechanism has been devised by Nishihara and Poggio (see Koch and Poggio, 1987) to account for the responses of tuned neurons to edges. It can be expanded upon to interpret the responses of these neurons to binocular contrast correlation and their typical insensitivity to monocular stimulation. With on this model, neurons receiving inputs evoked by opposite stimulus contrasts in the 2 eyes would respond with excitation to identical (brightbright or dark-dark depending on which contrast is excitatory) binocular matches (one eye excites, the other does not inhibit) and with suppression to bright-dark matches (the inhibitory input shunts off excitation). Moreover, neurons with this organization would give excitatory responses to binocularly correlated images (edges or random-dot patterns) and suppressive responses to uncorrelated images. Finally, these neurons would give little or no responses to monocular stimulation for if the inhibitory eye only is illuminated, it would have no visible synaptic effect at the cortical neuron, and if the excitatory eye is stimulated, its effects will be vetoed by shunting inhibition evoked from the not-seeing (dark!) eye. These response properties are typical of the tuned excitatory neurons, specifically of the tuned zero neurons (T0).

Those cells, on the other hand, at which the binocular synaptic inputs signal the same contrast in both eyes would be inhibited by bright-bright (or dark-dark) binocular matches and excitated by bright-dark matches. These neurons would give suppressive responses to binocularly correlated edges or random-dot patterns, activated by uncorrelated images, and generally have an unbalanced ocularity, responding with excitatory to stimulation of one eye only. These response properties are characteristics of the tuned inhibitory neurons (TI).

Anderson and Van Essen (1987) have considered a similar AND-NOT synaptic input arrangement for a model of a "shifter" mechanism that would maintain dynamically the disparity relation between binocular response fields by compensating for the sizable shifts in eye vergence (and associated changes in the relative positions of the fields in the 2 eyes). This model also rests on the massive and orderly inputs from the lateral geniculate nucleus to layer IVc of the striate area. Because of this common basis, the shifter model could be easily merged with the disparity model outlined above to account for the observed response behavior of stereo neurons in the alert, behaving macaque, at least for T0/TI type cells.

\section{$T N / T F$ and $N E / F A$ neurons}

No similar straightforward mechanisms can be offered for the binocular response of disparity selective neurons, whether ex- 
citatory tuned at larger disparities $\left(>0.2^{\circ}\right)$ or with reciprocal response profiles. On the other hand, a number of schemata can be put together for these cells based on synaptic arrangements of the binocular input subunits at the neuron's dendritic tree. These include both types of synaptic gates, shunting and summing, AND-NOT and AND-like. Since all logical operations can be synthesized simply in terms of these gates, all sort of arrangements using "synaptic logic" (Koch and Poggio, 1987) can be devised to construct disparity sensitivity profiles such as those experimentally observed for these neurons. Rall et al. (1967) have pointed out that shunting occurs between synapses located near each other, whereas linear summation takes place at widely separate locations. It is of interest to consider that for neurons with T0 or TI stereo properties, binocular interaction is clicited from subficlds (or receptive zoncs) with identical or nearly identical retinal topography, and thus are possibly projecting their inputs to adjacent synaptic terminals on the binocular neuron's dendrite. On the other hand, binocular interaction for tuned far and tuned near neurons, and even more so for the near and far neurons, takes place over a larger extent of retina, as shown by the wider range of effective disparities, and therefore between spatially separated retinal regions whose inputs may reach synapses at relatively distant locations on the neuron's dendritic tree. Whether these speculations can be tested experimentally is unclear at this time.

\section{References}

Anderson, C. H., and D. C. Van Essen (1987) Shifter circuits: A computational strategy for dynamic aspects of visual processing. Proc. Natl. Acad. Sci. USA 84: 6297-6301.

Baizer, J. S. (1982) Receptive field properties of V3 neurons in monkey. Invest. Ophthalmol. 23: 87-95.

Baizer, J. S., D. L. Robinson, and B. M. Dow (1977) Visual responses of area 18 neurons in awake, behaving monkey. J. Neurophysiol. 40: 1024-1037.

Barlow, H., C. Blakemore, and J. D. Pettigrew (1967) The neural mechanism of binocular depth discrimination. J. Physiol. (Lond.) 193: 327-342.

Bishop, P. O., and G. H. Henry (1971) Spatial vision. Annu. Rev. Psychol. 22: 119-161.

Bishop, P. O., G. H. Henry, and C. J. Smith (1971) Binocular interaction fields of single units in the cat striate cortex. J. Physiol. (Lond.) 216: 39-68.

Blasdel, G. G., and D. Fitzpatrick (1984) Physiological organization of layer 4 in macaque striate cortex. J. Neurosci. 4: 880-895.

Burkhalter, A., and D. C. Van Essen (1986) Processing of color, form and disparity information in visual areas VP and V2 of ventral extrastriate cortex in the macaque monkey. J. Neurosci. 6: 2327-2351.

Camarda, R. M., E. Peterhans, and P. O. Bishop (1985a) Spatial organizations of subregions in receptive fields of simple cells in cat striate cortex as revealed by stationary flashing bars and moving edges. Exp. Brain Res. 60: 136-150.

Camarda, R. M., E. Peterhans, and P. O. Bishop (1985b) Simple cells in cat striate cortex: responses to stationary flashing and to moving light bars. Exp. Brain Res. 60: 151-158.

Cragg, B. G. (1969) The topography of the afferent projections in the circumstriate visual cortex of the monkey studied by the Nauta method. Vision Res. 9: 733-747.

DeYoe, E. A., and D. C. Van Essen (1985) Segregation of afferent connections and receptive field properties of visual area V2 of the macaque. Nature 317: 58-61.

Dow, B. M., A. Z. Snyder, R. G. Vautin, and R. Bauer (1981) Magnification factor and receptive field size in foveal striate cortex of the monkey. Exp. Brain Res. 14: 213-228.

Dow, B. M., R. G. Vautin, and R. Bauer (1985) The mapping of visual space onto foveal striate cortex in the macaque monkey. J. Neurosci. 5: 890-902.
Emerson, R. C., M. C. Citron, W. J. Vaughn, and S. A. Klein (1987) Nonlinear directionally selective subunts in complex cells of cat striate cortex. J. Neurophysiol. 58: 33-65.

Felleman, D. J., and D. C. Van Essen (1987) Receptive field properties of neurons in area V3 of macaque monkey extrastriate cortex. J. Neurophysiol. 57: 889-920.

Ferster, D. (1981) A comparison of binocular depth mechanisms in area 17 and 18 of the cat visual cortex. J. Physiol. (Lond.) 311:623655.

Gattass, R., C. G. Gross, and J. H. Sandell (1981) Visual topography of V2 in the macaque. J. Comp. Neurol. 201: 519-539.

Glezer, V. D., T. A. Tsherbach, V. E. Gauselman, and V. M. Bondarko (1980) Linear and non-linear properties of simple and complex receptives fields in area 17 of the cat visual cortex. Biol. Cybernet. 37: 195-208.

Gonzalez, F., F. Krause, and G. F. Poggio (1986) Sensitivity of cortical visual neurons to binocular correlation of dynamic random-dot stereo patterns. Invest. Ophthalmol. Vis. Sci. (Suppl.) 27: 244.

Henry, G. H., P. O. Bishop, and J. S. Coombs (1969) Inhibitory and sub-liminal excitatory receptive fields of simple units in cat striate cortex. Vision Res. 9: 1289-1296.

Hubel, D. H., and M. S. Livingston (1987) Segregation of form, color, and stereopsis in primate area 18. J. Neurosci. 11: 3378-3415.

Hubel, D. H., and T. N. Wiesel (1962) Receptive fields, binocular interaction and functional architecture in the cat's visual cortex. J. Physiol. (Lond.) 160: 106-154.

Hubel, D. H., and T. N. Wiesel (1968) Receptive fields and functional architecture of monkey striate cortex. J. Physiol. (Lond.) 195: $215-$ 243.

Hubel, D. H., and T. N. Wiesel (1970) Cells sensitive to binocular depth in area 18 of the macaque monkey cortex. Nature 225: 41-42.

Hubel, D. H., and T. N. Wiesel (1977) Functional architecture of monkey visual cortex. Proc. R. Soc. London [Biol.] 198: 1-59.

Joshua, D. E., and P. O. Bishop (1970) Binocular single vision and depth discrimination. Receptive field disparities for central and peripheral vision and binocular interaction on peripheral single units in cat striate cortex. Exp. Brain Res. 10: 389-416.

Julesz, B. (1960) Binocular depth perception of computer-generated patterns. Bell Syst. Tech. J. 39: 1125-1162.

Julesz, B. (1971) Foundations of Cyclopean Perception, University of Chicago Press, Chicago.

Julesz, B., B. Breitmeyer, and W. Kropfl (1976) Binocular-disparitydependent upper-lower hemifield isotropy as revealed by dynamic random-dot stereograms. Perception 5: 129-141.

Koch, C., and T. Poggio (1987) Biophysics of computation: Neurons, synapses and membranes. In Synaptic Function, G. M. Edelman, W. E. Gall, and W. M. Cowan, eds., pp. 637-697, Wiley, New York.

Koch, C., T. Poggio, and V. Torre (1983) Nonlinear interactions in a dendritic tree: Localization, timing, and role in information processing. Proc. Natl. Acad. Sci. USA 80: 2799-2802.

Kulikowski, J. J., P. O. Bishop, and H. Kato (1981) Spatial arrangements of responses by cells in the cat visual cortex to light and dark bar and edges. Exp. Brain Res. 44: 371-385.

Livingstone, M. S., and D. H. Hubel (1984) Anatomy and physiology of a color system in the primate visual cortex. J. Neurosci. 4: 309356.

Livingstone, M. S., and D. H. Hubel (1987) Psychophysical evidence for separate channels for the perception of form, color, movement and depth. J. Neurosci. 11:3416-3468.

I und, J. S., A. E. Hendrickson, M. P. Ogren, and E. A. Tobin (1981) Anatomical organization of primate visual cortex area VII. J. Comp. Neurol. 202: 19-45.

Maske, R., S. Yamane, and P. O. Bishop (1984) Binocular simple cells for local stereopsis: Comparison of the receptive field organization for the two eyes. Vision Res. 24: 1921-1929.

Maske, R., S. Yamane, and P. O. Bishop (1985) Simple and B-cells in cat visual cortex. Complementarity of responses to moving light and dark bars. J. Neurophysiol. 53: 670-685.

Maske, R., S. Yamane, and P. O. Bishop (1986a) Stereoscopic mechanisms: Binocular responses of the striate cells of cats to moving light and dark bars. Proc. R. Soc. London [Biol.] 229: 227-256.

Maske, R., S. Yamane, and P. O. Bishop (1986b) End-stopped cells and binocular depth discrimination in the striate cortex of cats. Proc. R. Soc. London [Biol.] 229: 257-276.

Maunsell, J. H. R., and D. C. Van Essen (1983) Functional properties of neurons in middle temporal visual area of the macaque monkey. 
II. Binocular interaction and sensitivity to binocular disparity. J. Neurophysiol. 49: 1148-1167.

Motter, B. C., and G. F. Poggio (1984) Binocular fixation in the rhesus monkey: Spatial and temporal characteristics. Exp. Brain Res. 54: 304-314.

Movshon, J. A., I. D. Thompson, and D. J. Tolhurst (1978) Receptive field organization of complex cells in the cat's striate cortex. J. Physiol. (Lond.) 283: 79-99.

Mullikin, W. H., J. J. Jones, and L. A. Palmer (1984a) Receptive-field properties and laminar distribution of X-like and Y-like simple cells in cat area 17. J. Neurophysiol. 52: 350-371.

Mullikin, W. H., J. J. Jones, and L. A. Palmer (1984b) Periodic simple cells in cat area 17. J. Neurophysiol. 52: 372-387.

Nikara, T., Bishop, P. O., and J. D. Pettigrew (1968) Analysis of retinal correspondence by studying receptive fields of binocular single units in cat striate cortex. Exp. Brain Res. 6: 353-372.

O'Shca, R. P., and R. Blakc (1986) Depth without disparity in randomdot stereograms. Invest. Ophthalmol. Vis. Sci. (Suppl.) 27: 180.

Poggio, G. F. (1980) Neurons sensitive to dynamic random-dot stereograms in areas 17 and 18 of the rhesus monkey cortex. Soc. Neurosci. Abstr. 6: 672.

Poggio, G. F. (1984) Processing of stereoscopic information in monkey visual cortex. In Dynamic Aspects of Neocortical Function, G. M. Edelman, W. E. Gall, W. M. Cowan, eds., pp. 613-635, Wiley, New York.

Poggio, G. F. (1985) Cortical mechanisms of stereopsis. Invest. Ophthalmol. Vis. Sci. 26: 133.

Poggio, G. F., and B. Fischer (1977) Binocular interaction and depth sensitivity in striate and prestriate cortex of behaving rhesus monkeys. J. Neurophysiol. 40: 1392-1407.

Poggio, G. F., and T. Poggio (1984) The analysis of stereopsis. Annu. Rev. Neurosci. 7: 379-412.

Poggio, G. F., and W. H. Talbot (1981) Neural mechanisms of static and dynamic stereopsis in foveal striate cortex of rhesus monkeys. J. Physiol. (Lond.) 315: 469-492.

Poggio, G. F., R. W. Doty, and W. H. Talbot (1977) Foveal striate cortex of behaving monkey: Single-neuron responses to square-wave gratings during fixation of gaze. J. Neurophysiol. 40: 1369-1391.

Poggio, G. F., F. Gonzalez, and F. Krause (1985a) Binocular correlation system in monkey visual cortex. Soc. Neurosci. Abstr. 11: 17.

Poggio, G. F., B. C. Motter, S. Squatrito, and Y. Trotter (1985b) Responses of neurons in visual cortex (V1 and $\mathrm{V} 2$ ) of the alert macaque to dynamic random-dot stereograms. Vision Res. $25: 397-406$.

Rall, W. (1964) Theoretical significance of dendritic trees for neuronal input-output relations. In Ncural Theory and Modcling, R. Reiss, ed., pp. 73-97, Stanford University Press, Stanford, CA.

Rall, W., R. E. Burke, T. G. Smith, P. G. Nelson, and K. Frank (1967) Dendritic location of synapses and possible mechanisms for the monosynaptic EPSP in motoneurons. J. Neurophysiol. 30: 11691193.

Schiller, P. H., B. L. Finlay, and S. F. Volman (1976a) Quantitative studies of single-cell properties in monkey striate cortex. I. Spatiotemporal organization of receptive fields. J. Neurophysiol. 39: 12881319.

Schiller, P. H., B. L. Finlay, and S. F. Volman (1976b) Quantitative studies of single-cell properties in monkey striate cortex. II. Orientation specificity and ocular dominance. J. Neurophysiol. 39: 13201333.

Shipp, S., and S. Zeki (1985) Segregation of pathways leading from area V 2 to areas V4 and V 5 of macaque monkey visual cortex. Nature 315: $322-325$

Skottun, B. C., and R. D. Freeman (1984) Stimulus specificity of binocular cells in cat's visual cortex: Ocular dominance and the matching of left and right eyes. Exp. Brain Res. 56: 206-216.

Tootell, R. B. H., M. S. Silverman, R. L. DeValois, and G. H. Jacobs (1983) Functional organization of the second cortical visual area in primates. Science 220:737-739.

Tyler, C. W., and B. Julesz (1976) The neural transfer characteristics (Neurontropy) for binocular stochastic stimulation. Biol. Cybernet. 23: 33-37.

Tyler, C. W., and B. Julesz (1978) Binocular cross-correlation in time and space. Vision Res. 18: 101-105.

Van Essen, D. C., and S. M. Zeki (1978) The topographic organization of rhesus monkey prestriate cortex. J. Physiol. (Lond.) 277: 193-226.

Van Essen, D. C., W. T. Newsome, and J. L. Bixby (1982) The pattern of interhemispheric connections and its relationship to extrastriate visual areas in the macaque monkey. J. Neurosci. 2: 265-283.

Van Essen, D. C., W. T. Newsome, and J. H. R. Maunsell (1984) The visual field representation in striate cortex of the macaque monkey: Asymmetries, anisotropies, and individual variability. Vision Res. 24: 429-448.

Van Essen, D. C., W. T. Newsome, J. H. R. Maunsell, and J. L. Bixby (1986) The projections from striate cortex (V1) to visual areas V2 and V 3 in the macaque monkey: Asymmetries, areal boundaries, and patchy connections. J. Comp. Neurol. 244: 451-480.

Wheatstone, C. (1838) Contributions to the physiology of vision. Part the first. On some remarkable, and hitherto unobserved, phenomena of binocular vision. Phil. Trans. R. Soc. 2: 371-393.

Wolbarsht, M. L., E. F. MacNichol, Jr., and H. G. Wagner (1960) Glass insulated platinum microelectrode. Science 132: 1309-1310.

Zeki, S. M. (1969) Representation of central visual fields in prestriate cortex of monkey. Brain Res. 14: 271-291.

7eki, S. M. (1978a) The third visual complex of rhesus monkey prestriate cortex. J. Physiol. (Lond.) 277: 245-272.

Zeki, S. M. (1978b) Uniformity and diversity of structure and function in rhesus monkey prestriate visual cortex. J. Physiol. (Lond.) 277: 273-290.

Zeki, S. M., and D. R. Sandeman (1976) Combined anatomical and electrophysiological studies on the boundary between second and third visual areas of rhesus monkey cortex. Proc. R. Soc. London [Biol.] 194: 555-562. 